\title{
Fiber deposition models in two and three spatial dimensions
}

\author{
N. Provatas a, M. Haataja ${ }^{\text {b }}$ J. Asikainen ${ }^{\text {c,d }}$, S. Majaniemi ${ }^{\text {c,d }}$, M. Alava ${ }^{\text {d, }}$ \\ T. Ala-Nissila ${ }^{\mathrm{c}, \mathrm{d}, \mathrm{e}, *}$ \\ a Departments of Physics and Mechanical Engineering, University of Illinois at Urbana-Champaign, Loomis Laboratory of Physics, \\ 1110 West Green Street, Urbana, IL 61801-3080, USA \\ ${ }^{\mathrm{b}}$ Centre for the Physics of Materials, Department of Physics, McGill University, Rutherford Building, 3600 rue University, \\ Montréal, Qué., Canada H3A $2 T 8$ \\ ${ }^{\mathrm{c}}$ Helsinki Institute of Physics, PO Box 9 (Siltavuorenpenger 20 C), FIN-00014 University of Helsinki, Helsinki, Finland \\ ${ }^{\mathrm{d}}$ Laboratory of Physics, Helsinki University of Technology, PO Box 1100, FIN-02150 HUT, Espoo, Finland \\ ${ }^{\mathrm{e}}$ Department of Physics, Brown University, Providence, Rhode Island 02912, USA
}

\begin{abstract}
We review growth, percolation, and spatial correlations in deposition models of disordered fiber networks. We first consider 2D models with effective interactions between the deposited particles represented by simple parametrization. In particular, we discuss the case of single cluster growth, growth of uniformly random networks, and flocculated networks with nontrivial spatial correlations. We also consider a 3D deposition model of flexible fibers that describes the growth of multilayer structures of disordered networks. We discuss the statistical properties of such structures, transport of fluid through the network, and the asymptotics of growth in the limit of infinite thickness. (C) 2000 Elsevier Science B.V. All rights reserved.
\end{abstract}

Keywords: Deposition models; Fiber networks; Spatial correlations; Paper structure; Continuum percolation

\section{Introduction}

There are many phenomena in nature that can be viewed as deposition processes where various transport mechanisms bring particles to a surface. These include deposition of colloidal, polymer and fiber particles [1-8]. In some cases, such deposition phenomena involve particles whose size is large compared with the their mutual inter-

\footnotetext{
* Corresponding author.

E-mail addresses: provatas@uiuc.edu (N. Provatas),
} alanissi@csc.fi (T. Ala-Nissila) action range, and so that the main deposition mechanism is due to particle exclusion. Among the most studied models in this class of phenomena are the random and cooperative sequential adsorption models [1,2]. There particles are deposited on a surface and either stick or are rejected according to certain exclusion rules, with a maximum coverage (the 'jamming limit') less than the close-packing limit. These types of models should be contrasted with the case of multilayer surface growth $[1,6,7]$, where the main focus is in the asymptotic behavior of the growing surface in the continuum limit [9]. 
A particularly interesting and challenging example involving particle deposition can occur in the case of colloidal suspensions. For some such systems, the inter-particle repulsion is strong enough to prevent multilayer growth [3]. However, the existence of dispersion forces can cause the particles to flocculate, or aggregate, and to precipitate out of the suspension [7,10]. For larger particles or clusters of particles, gravity often induces sedimentation out of the suspension. Experiments reveal that sedimentation produces nontrivial spatial structures [11]. A full microscopic treatment of sedimentation is a formidable task, however [12].

An interesting deposit forms during the preparation of laboratory-made paper. Such paper is manufactured from a suspension, out of which fibers filtrate onto a wire mesh, leaving behind a disordered fiber network [4]. Recently, sheets of such paper with varying basis weights (areal mass densities) ranging from 6.6 to $120 \mathrm{~g} \mathrm{~m}^{-2}$ were examined using the beta-radiography technique [13]. Fig. 1 shows an example of a typical radiograph from a sheet of basis weight of $80 \mathrm{~g} \mathrm{~m}^{-2}$.

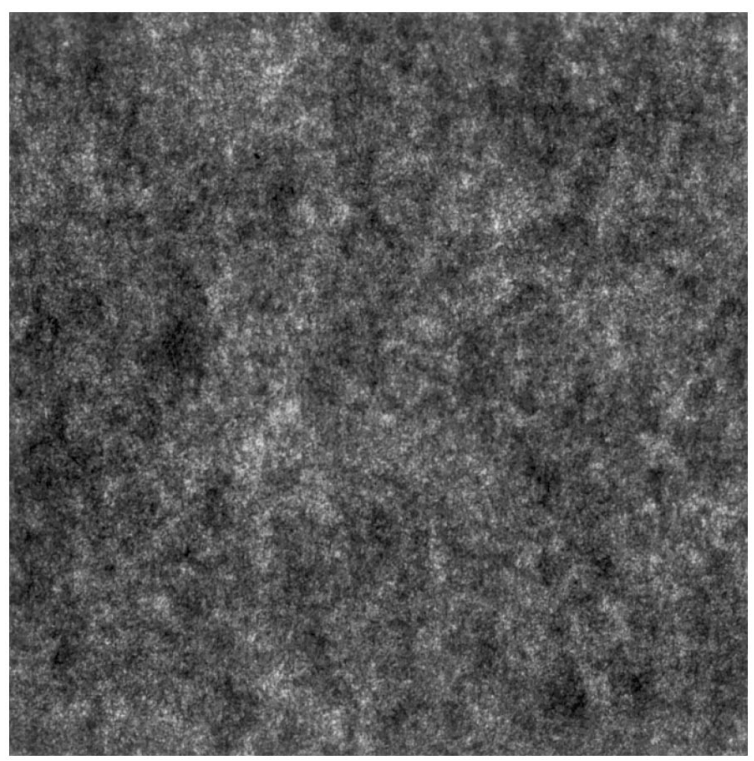

Fig. 1. A $10 \times 10 \mathrm{~cm}^{2}$ sample radiograph of a paper sheet with basis weight $80 \mathrm{~g} \mathrm{~m}^{-2}$. The grayscale has ten shades, starting from zero basis weight and increasing with steps of $14.5 \mathrm{~g}$ $\mathrm{cm}^{-2}$.
To study spatial correlations, the two-point density correlation function

$G(\vec{r})=\langle[m(\vec{x})-\bar{m}][m(\vec{x}+\vec{r})-\bar{m}]\rangle$

was calculated, where $m(\vec{x})$ is the local mass density, $\bar{m}$ its average and $\langle\cdot\rangle$ an average over each sheet. Fig. 2 shows $G(r)$ for four basis weights on a $\log -\log$ plot. It was found that for all the data there is a regime where $G(r)$ is well approximated by

$G(r) \simeq r^{-\alpha}$,

where $\alpha=0.37 \pm 0.07$. The remarkable finding was that for low basis weight sheets, the power law behavior extends up to more than ten times the average fiber length. Thus, the properties of $G(r)$ reflect the complex processes occurring during paper-making, such as fiber flocculation in suspension, sedimentation and filtration that lead to nontrivial spatial correlations. A microscopic understanding of this is currently missing. Because of this, phenomenological deposition models may be useful in studying how various effective interactions influence the mass density distributions of the consequent deposits.

In addition to their practical applications, 2D deposition models have been the topic of intense study in their own right. In particular, they have been extensively studied in the context of continuum percolation theory [14-27]. These models have included both uniformly random networks of various objects as well as some that include hard and soft-core interactions between the constituent particles. The quantity of central importance in these studies is the percolation threshold or critical particle density, which for permeable objects can be related to the excluded volume of the particles [20]. This quantity depends on the geometrical shape of the deposited particles as well as on interactions between them in a nontrivial way.

In reality, disordered fiber networks such as paper are actually three-dimensional, and thus many properties of such networks need appropriate modeling. For this purpose, it is important to understand the crossover from 2- to 3D behavior by studying models which can grow networks of finite thickness that have pores in the direction 


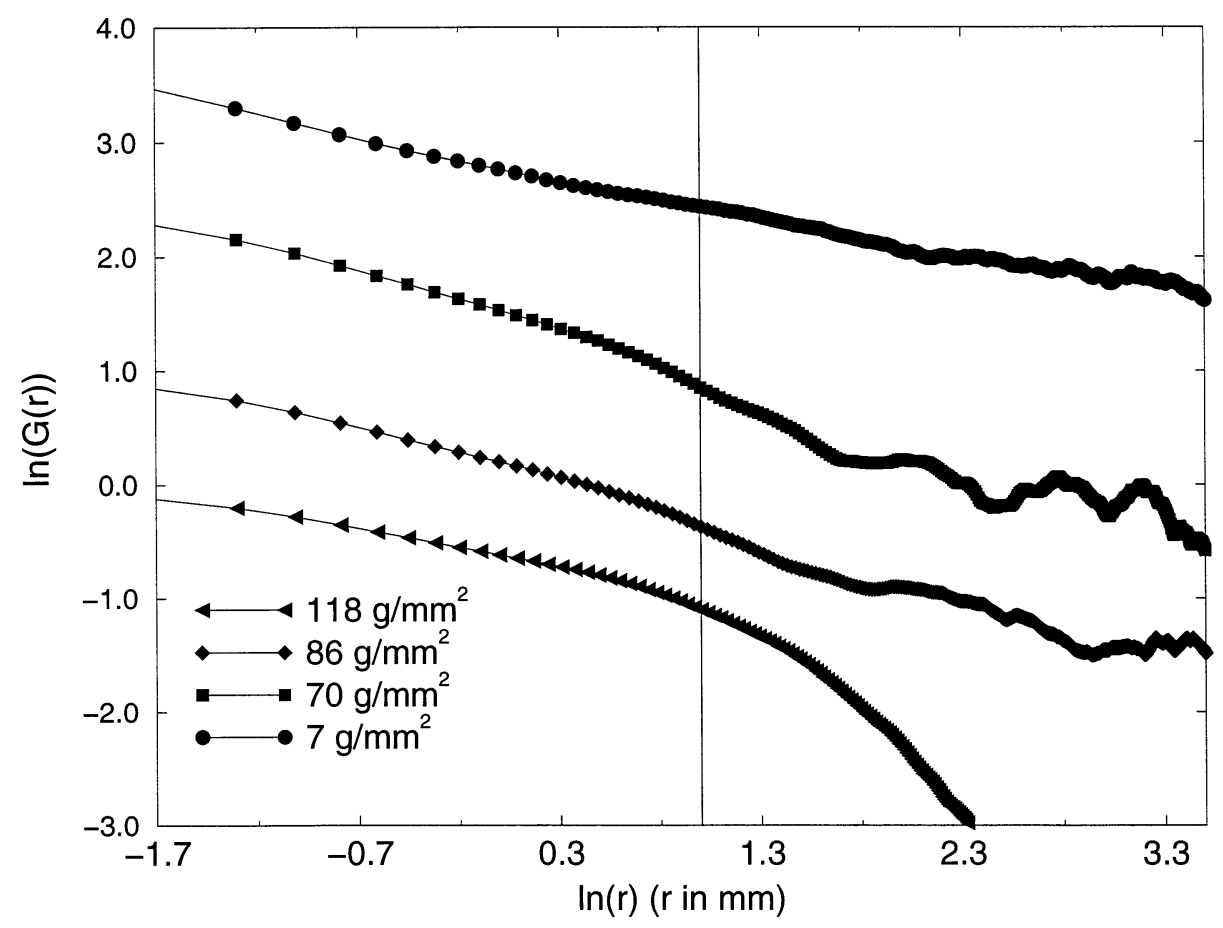

Fig. 2. A $\log -\log$ plot of the correlation function $G(r)$ for four paper sheets of basis weights $7.2,70.4,86.3$, and $118.9 \mathrm{~g} \mathrm{~m}^{-2}$ (from top to bottom). The vertical line indicates the average fiber length $\lambda=2.7 \mathrm{~mm}$. The curves have been shifted for clarity. For the lowest densities, the power law extends up to about 14 times the fiber length.

perpendicular to the deposit plane [5]. Such models are important when e.g. examining transport processes in fiber networks [28].

In this review, we address the fundamental properties of deposition phenomena using simple two- and three-dimensional deposition models. In particular, in 2D we examine simple deposition models with effective interactions between the deposited objects. Using such models, we study the generic structural properties of disordered networks of rectangles, disks and needles. We compare and contrast the properties of disordered needle networks with those of real paper sheets. To better understand the three-dimensional nature of fiber networks, we review the properties of a simple 3D deposition model that takes into account the finite flexibility of fibers. This creates a pore structure along with the growth of the deposit, and highlights how the geometry begins to differ from the $2 \mathrm{D}$ case. With a $3 \mathrm{D}$ model, it also becomes possible to discuss interesting issues such as transport of liquid through a fiber network, and the asymptotics of surface roughening.

\section{Fiber deposition models}

In this section, we review the properties of $2 \mathrm{D}$ deposition models which have been introduced and discussed in detail in Refs. [29-31]. In the first model, the tendency of the deposited particles to form clusters is taken into account through an effective attractive interaction. The effect of clustering is in part motivated by the tendency of fibers to form clusters in paper-making, a property known as flocculation. Hence, we have coined this model the 'flocculation model'. Note that this implies an effective description of clustering, since in real deposition phenomena flocs form in the suspension before ending up to the deposit. In the second case, there is an effective repulsive interaction between the deposited objects, that in the 
limit of a strict non-overlap condition leads to the random sequential adsorption (RSA) case. This model is called the 'rejection model'. We review the growth dynamics, percolation thresholds and spatial correlations in the two models for disordered networks of needles, fibers of various aspects ratios, and discs.

\subsection{Flocculation model}

A simple way of enhancing cluster formation of deposited objects in 2D networks is to favor the accumulation of deposited objects in areas where deposition has already taken place. This is achieved by the following deposition rules. Objects are deposited in a two-dimensional system of linear dimension $L$. Both the orientation and the spatial coordinate of the objects are drawn out of a uniformly random distribution. If a deposited object lands on another object already on the surface, the attempt is always accepted. However, if it lands on empty space the attempt is accepted only with a given probability $p$, called the acceptance probability.

In the limit $p=1$ the model reduces to the extensively studied case of a uniformly random network [4,14-18,24,32]. However, for $p<1$ there are effective interactions between the particles that tend to enhance cluster formation. In particular, for the extreme case of $p=0$, only particles that touch each other are accepted, leading to a

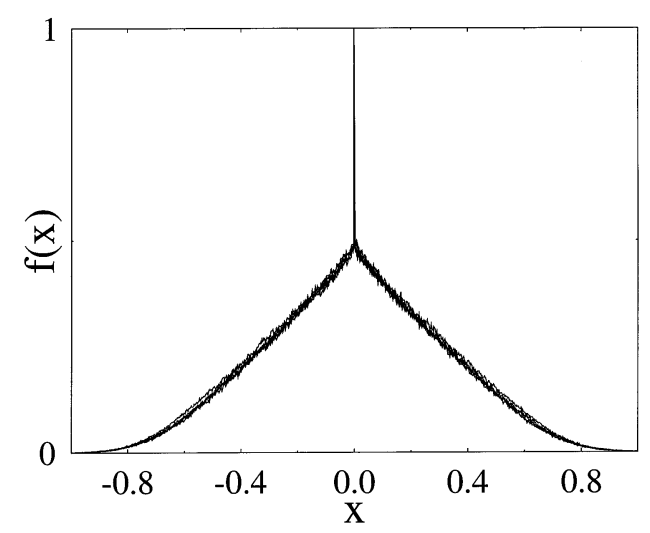

Fig. 3. The scaling function of Eq. (4)) $f(x)$ vs. $x=r / R(N)$ for $N=1500,2000,2500,3000$ and 4000 fibers, with an aspect ratio of $50 / 1$. single cluster (growing from an initial seed object).

The important statistical properties of these individual clusters are the mean radius $R(N)$, the radial mass density $\rho(r, N)$, and the roughness $W(N)$ of the cluster edge [33,29], where $N$ denotes the number of objects in the cluster. The statistically spherically symmetric radius $R(N)$ was shown to follow the growth law $[29,33]$

$R(N)=B N^{1 /(d+1)}$,

for $N \gg 1$, where $d$ is the spatial dimension of the deposit. In particular, for 2D planar deposits the growth follows a $1 / 3$ law. The constant $B$ depends only on the object shape. In the same limit, $\rho(r$, $N)$ is given by

$\rho(r, N)=\frac{3 K}{\pi B^{3}} R(N) f(r / R(N))$,

where $K$ is the mass flux per unit area, and the scaling function $f(r / R(N))=1-r / R(N)$ in a mean-field approximation [29]. Fig. 3 shows a plot of the scaling function $f(x)$ for fiber clusters for five different values of $N$. The vertical bar at the origin, from the cusp up, is an artifact arising due to the fact that the seed fibre always intersects the origin. As a result, statistical averaging of the origin will always have an offset. An interesting feature not captured by the mean-field approximation of $f(x)$ is that the cluster edge is rough. Its width was shown to follow, in 2D, the same growth law as $R(N)$, i.e. $W(N)=\langle(\tilde{R}(N)-$ $\left.R(N))^{2}\right\rangle^{1 / 2} \sim N^{1 / 3}$, since $W / R=$ const.

We next examine the percolation properties of the flocculation model for $p>0$. Typical configurations of needle deposits are shown in Fig. 4. When enough objects are deposited in a finite system, its edges become connected and a continuum percolation transition [20,34] occurs. In the thermodynamic limit when $N \rightarrow \infty$ and $L \rightarrow \infty$, with $\eta=N / L^{2}=$ const., percolation occurs at a critical number density $\eta_{\mathrm{c}}$. For the case of the flocculation model, a detailed exposition of its percolation properties has been given in Refs. $[29,35]$. These works examined percolation in networks of needles, fibers and discs. The results are summarized here. 

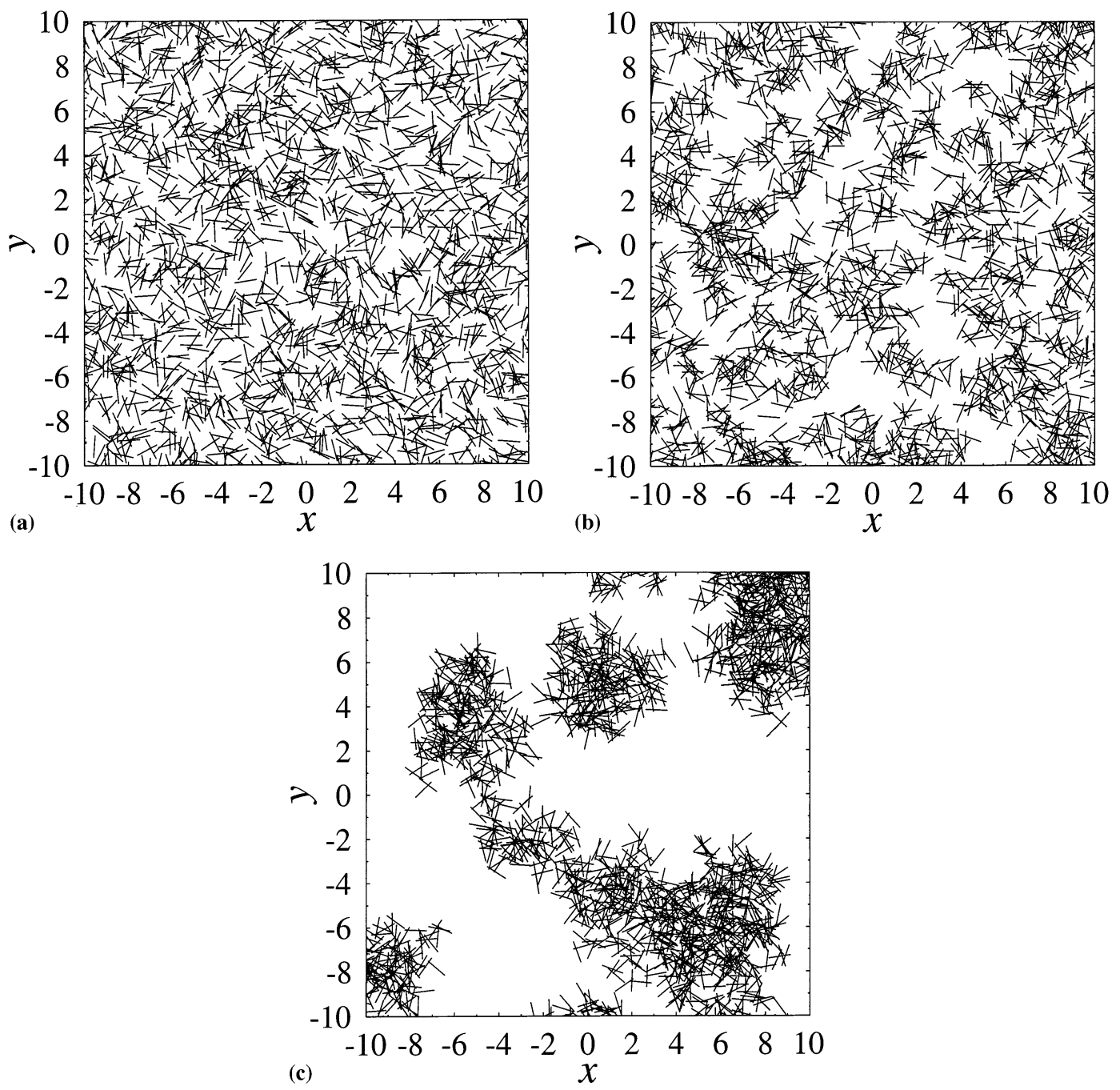

Fig. 4. Snapshots of configurations of disordered networks as generated from the flocculation model with $N=2000$ needles of unit length, with (a) $p=1$ (a uniformly random network); (b) $p=0.1$, and (c) $p=0.001$.

There are two interesting regimes in the percolation properties of the model. The first occurs when $p \rightarrow 0$, and the second, when $p \rightarrow 1$. An illustration of the percolation threshold $\eta_{\mathrm{c}}(p)$ for needles is shown in Fig. 5; results for fibers and discs are qualitatively similar [29,35]. The behavior of $\eta_{\mathrm{c}}$ for $p \ll 1$ can be understood using mean field arguments [29] that predict a universal divergence of $\eta_{\mathrm{c}}(p)$ in the limit $p \rightarrow 0$ as

$\eta_{\mathrm{c}}(p) \propto p^{-1 / 2}$
The divergence results because when a single connected cluster grows, percolation occurs when the cluster radius $R=L$, which means that the total number of deposited objects in the cluster is $N_{\mathrm{c}} \propto L^{3}$. As a result, $\eta_{\mathrm{c}}=N_{\mathrm{c}} / L^{2} \propto L \rightarrow \infty$ in the thermodynamic limit.

In the opposite limit when the deposit is close to a uniformly random network, i.e. $p$ close to 1 , $\eta_{\mathrm{c}}(p)$ was found to increase linearly as

$\eta_{\mathrm{c}}(p)=A p+B$ 
where $A$ and $B$ are constants. This can be understood by the following mean-field argument. Let us imagine a uniformly random network (i.e. $p=$ $1)$ at its percolation threshold $\eta_{\mathrm{c}}(p=1)$. One effect of decreasing $p$ is to remove those fibers that are not connected to the percolating cluster, while the backbone of the percolating cluster can be assumed to stay intact. It can be shown [29] that the number of fibers removed is proportional to $1-$ $p$, and therefore leads to a linear change in $\eta_{\mathrm{c}}(p)$ It should be stressed that this behavior is found for all object geometries studied. It is interesting to note that due to the competition between clustering and uniform filling, there is an 'optimal' way of forming the cluster at some intermediate value of $0<p<1$, where the critical density is minimal.

\subsection{Rejection model}

The case of the 'rejection model' is opposite to that of the flocculation model, in that an effective particle-particle repulsion is generated. In this case, if a deposited object lands on empty space, the attempt is always accepted. However, if it lands on another object already on the surface,

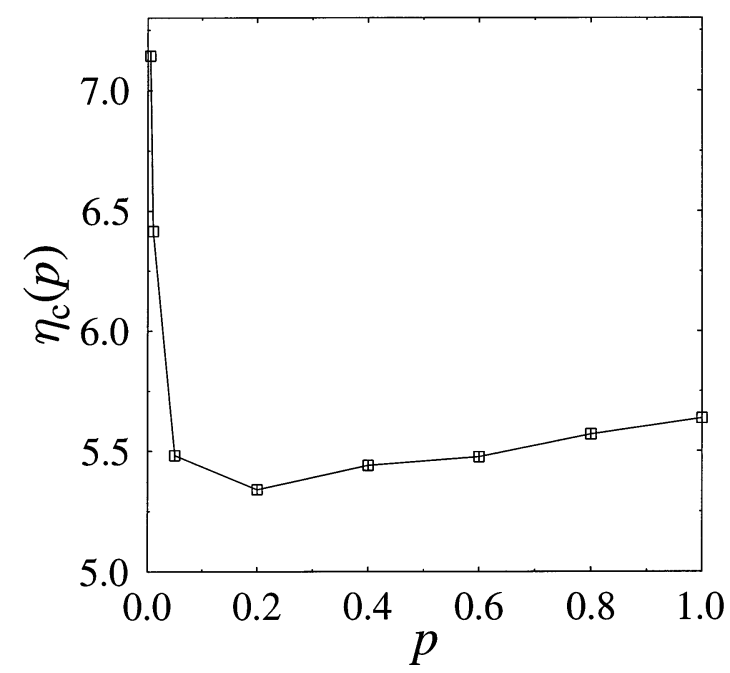

Fig. 5. The critical percolation threshold $\eta_{\mathrm{c}}(p)$ vs. $p$ for a network of needles of unit length, extrapolated to $L \rightarrow \infty$. The error bars are of the same order as the size of the points. the attempt is rejected with a probability $0 \leq q \leq$ 1.

In the limit $q=0$ the rejection model produces uniformly random networks, just as the flocculation model does for $p=1$. However, the limit $q=1$ is more interesting, since only particles that do not touch each other are accepted. This is the well known case of random sequential adsorption (RSA) models [1]. In this case, percolation with connectivity defined through particle overlap is never achieved, and for particles having a finite area the deposition terminates to a finite density called the 'jamming limit'. However, if one defines the connectivity rule to require only that particles are within a certain distance of each other to be in the same cluster it is possible to have a percolating cluster [36].

Typical configurations of needles generated by the rejection model are shown in Fig. 6 for various values of $q$, and can be contrasted to those of Fig. 4. In Ref. [30], the percolation properties of the model were studied in detail analytically, and numerically. The corresponding critical densities for needles are shown in Fig. 7. Using arguments similar to the case of the flocculation model, it was shown than for $q \ll 1$, the critical density increases linearly as

$\eta_{\mathrm{c}}(q)=A^{\prime} q+B^{\prime}$

where $A^{\prime}$ and $B^{\prime}$ are constants. This result follows because for weak repulsion, there are added nonintersecting particles in the system as compared to a uniformly random network.

In the other limit, the critical density in Fig. 7 shows an apparent divergence when the RSA limit is approached, since the deposited needles have no width. It was indeed shown that the expected behavior for $q \rightarrow 1$ is given by

$\eta_{\mathrm{c}}(q) \propto(1-q)^{-\gamma}$,

where $\gamma \approx 0.32$ is known numerically only [37]. In the case of fibers and other objects with a finite area, however, there is no divergence of $\eta_{\mathrm{c}}(q=1)$, since a finite jamming limit $\eta_{\mathrm{j}}$ will be reached. An upper limit for the percolation threshold can thus be approximately given by $\eta_{c}(q=1) \leq \eta_{\mathrm{c}}(q=0)+$ $\eta_{\mathrm{j}}$. 

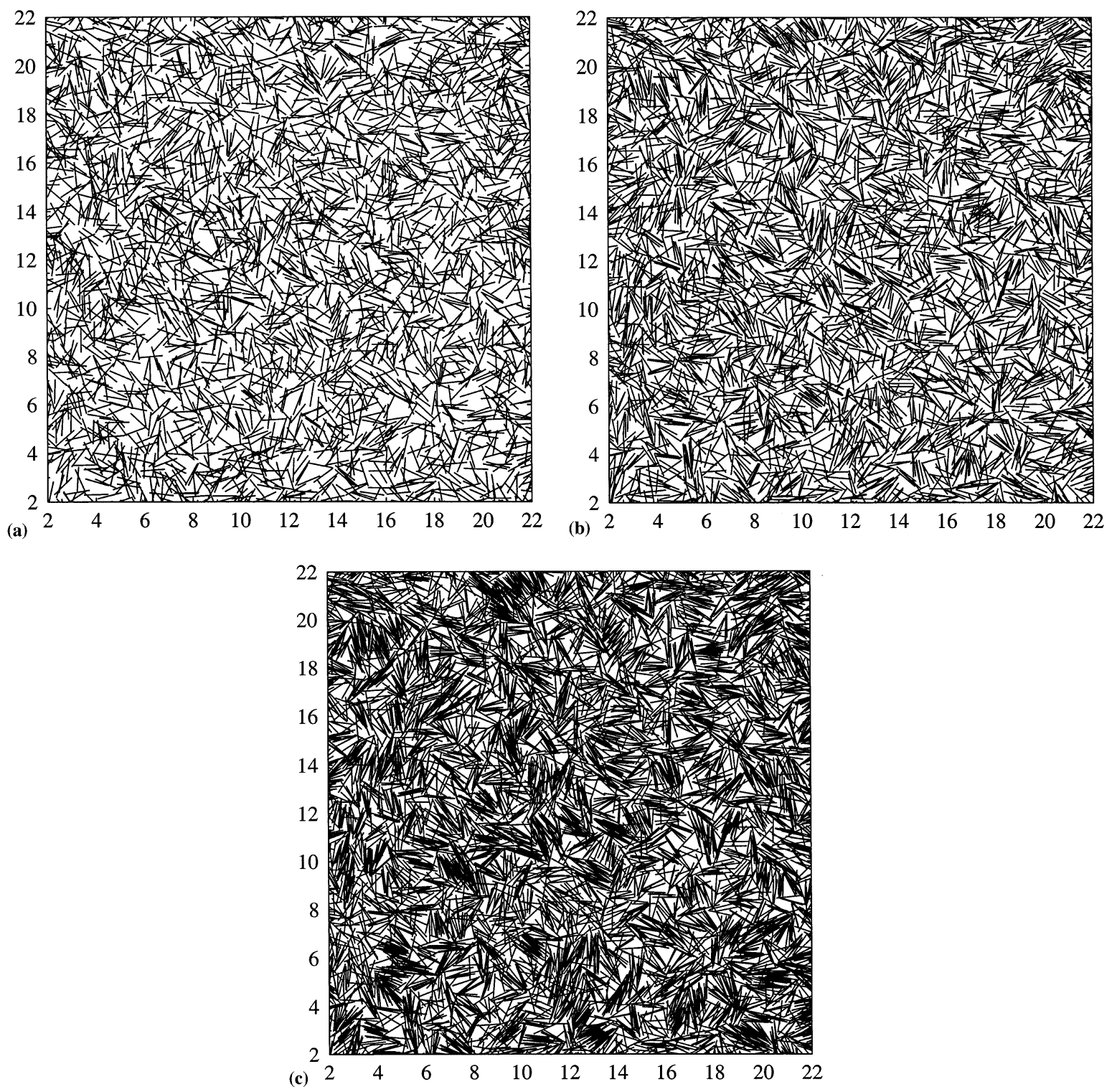

Fig. 6. Snapshots of configurations of disordered networks as generated from the rejection model with needles of unit length, with (a) $q=0.9, N \approx 2800$; (b) $q=0.99, N \approx 3600$, and (c) $q=0.999, N \approx 6300$.

\subsection{Spatial correlations}

An interesting question concerns the nature of spatial correlations induced by the effective interactions in the $2 \mathrm{D}$ deposition models. We review here the properties of the two-point density correlation functions, and the pair distribution func- tions in networks produced by the flocculation and rejection models.

\subsubsection{Two-point mass density correlations}

In Ref. [29], a general expression was derived for the two-point mass density correlation function of Eq. (1). This form of $G(r)$ was shown to 
decompose into two contributions. The first part is due to the effect of the self-correlation of single deposited objects. The second term comes from the effective spatial interactions between the deposited objects. In the simplest case of uniformly random deposits, this second term vanishes and $G(r)$ depends on the particle geometry only.

In Fig. 8(a) and (b) we show a series of correlation functions $G(r)$ for the case of fiber networks as calculated numerically from the flocculation and rejection models, respectively. The form of $G(r)$ is rather well approximated by

$G(r) \sim r^{-\alpha(N)}$, for $0<r<\Lambda(N)$,

where $\Lambda$ is an effective cutoff which depends on the probabilities $p$ and $q$ in the two models. For the flocculation model, the effective exponent $\alpha(N, p)$ (with $p<1$ ) attains a minimum as $N$ increases, while exactly the opposite is true for $\alpha(N, q)$ in the rejection model $(q>0)$. The cutoffs behave differently also, with $\alpha(N, p)$ being smallest when $\Lambda(N, p)$ for the flocculation model is maximal, while $\alpha(N, q)$ is largest when $\Lambda(N, q)$ is smallest.

Fig. 9(a) shows a series of effective exponents $\alpha(N, p)$ versus $N$ for the flocculation model. The behaviour of $\alpha$ can be understood as a competition between individual fiber clusters and uniformly random fibers, both of which coexist for $0<p<1$. The approach of the correlation exponent toward $\alpha \approx 1$ as $N \rightarrow \infty$ is caused because in this limit the effect of $p$ gradually becomes unimportant [29], and the network tends towards a uniformly random one. On the other hand, the initial decrease of the effective $\alpha(N, p)$ for small values of $N$ (as well as the increase of the range of the corresponding power law regime) is due to the growth of essentially independent fiber clusters.

A better understanding of the low $N$ behaviour of $\alpha(N, p)$, as well as the increase in the power law regime, can be obtained by considering the particular case of $p=0$. In this limit, only a single cluster emerges. It was found that $G(r)$, as aver-

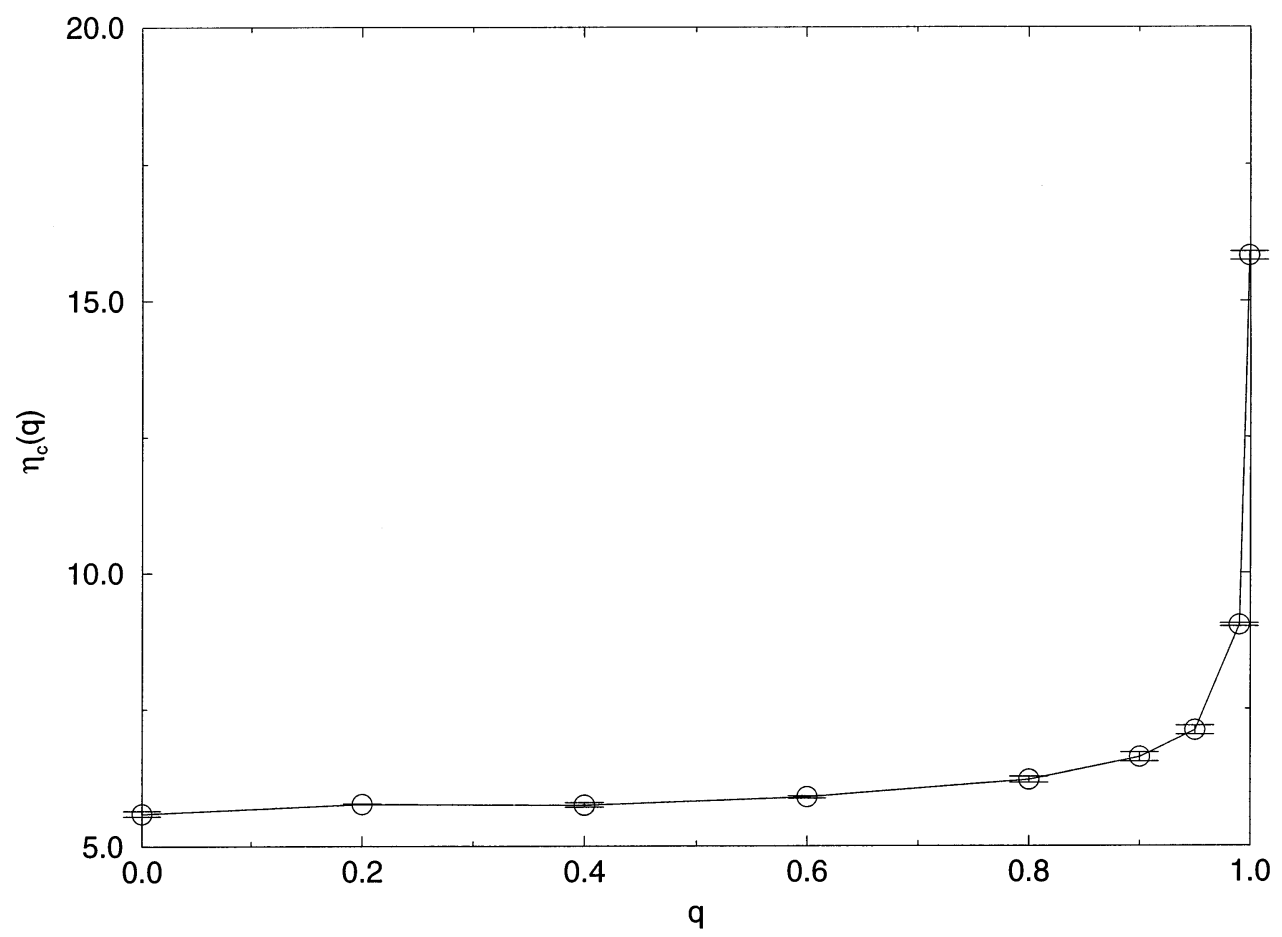

Fig. 7. The critical percolation threshold $\eta_{\mathrm{c}}(q)$ vs. $q$ for a network of needles of unit length, extrapolated to $L \rightarrow \infty$. 

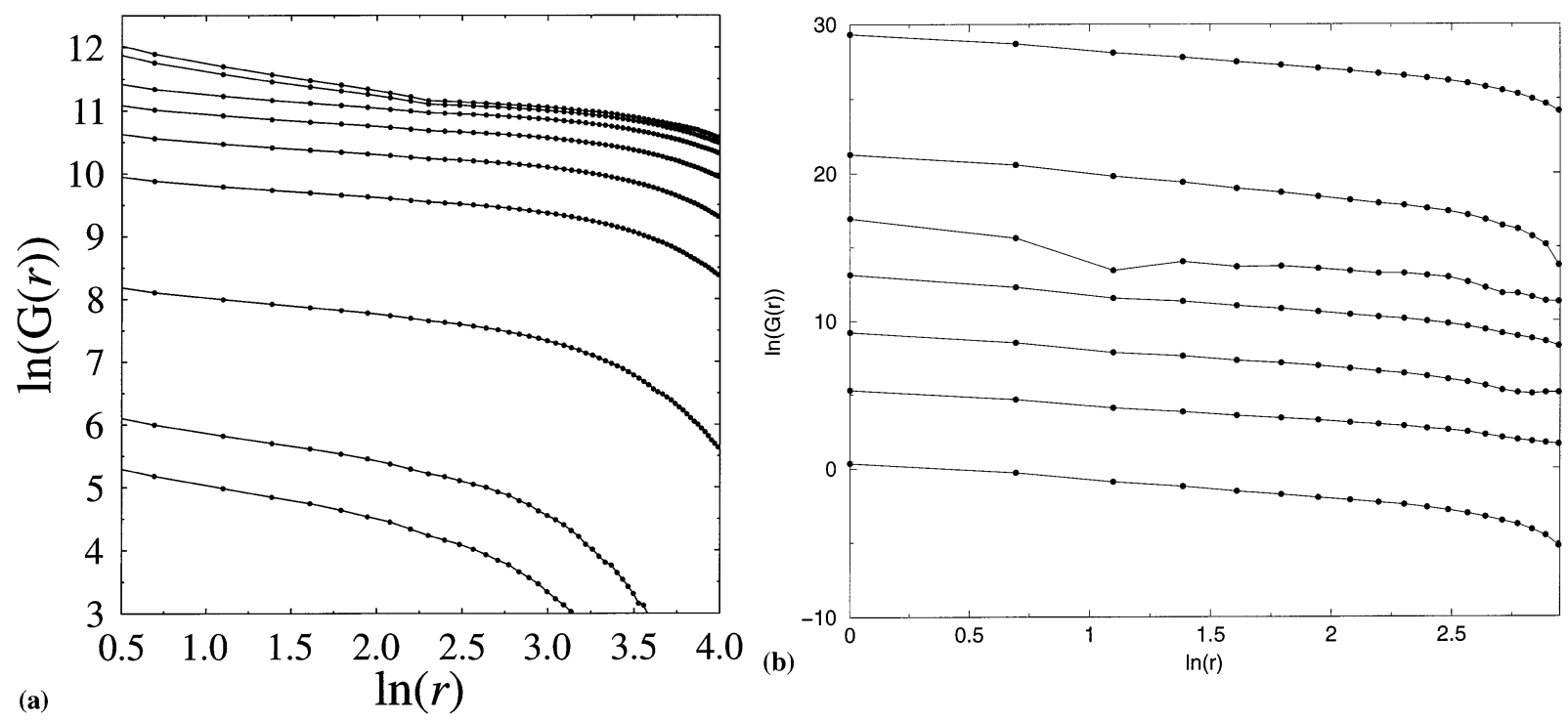

Fig. 8. A plot of $\ln (G(r))$ vs. $\ln (r)$ for fibers of length $\lambda=20$ and width $\omega=1$, for (a) $p=0.001$ in the flocculation model, and (b) $q=0.999$ in the rejection model. From bottom to top for (a) $N$ increases with $N=25,50,300,1500,3000,5000,8000,20000,25000$; for (b) $N=25,50,300,1500,3000,5000,10000$. The system size $L=400$, and $N_{\mathrm{c}} \approx 5000$ for the flocculation model, and $N_{\mathrm{c}} \approx 2000$ for the rejection model.
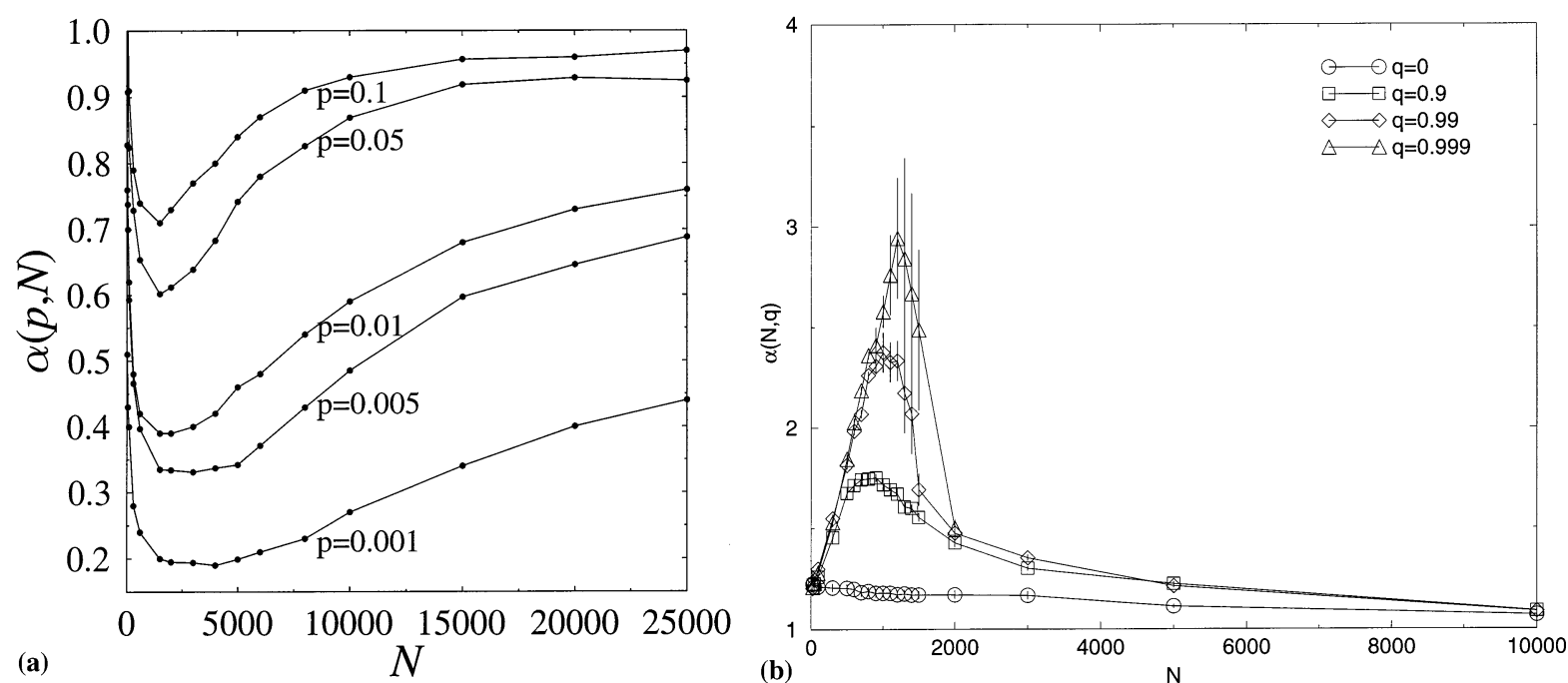

Fig. 9. A plot of the effective exponent (a) $\alpha(N, p)$ (flocculation model), and (b) $\alpha(N, q)$ (rejection model) vs. $N$ for various values of $p$ and $q$. The aspect ratio is $L / \lambda / \omega=400 / 20 / 1$.

aged over an ensemble of clusters of size $N$, is also well approximated by a power law form, and as $N$ increases, $\alpha$ decays to a fixed value of about 0.05 .
Moreover, the range of the power law is proportional to the radius of the cluster, with a constant of proportionality of about 0.7 [29]. The exponent 
$\alpha$ reaches its saturated value approximately at the same value of $N$ where the scaling laws of single cluster growth start to become valid.

In Fig. 9(b) we show the effective exponents $\alpha(N, q)$ versus $N$ for the rejection model, for various values of $q$. Behavior rather different from that of the flocculation model can be seen. For increasing $q$, the fibers tend to pack together and form locally aligned structures. This is reflected in anticorrelations of the mass density at distances just larger than the fiber width, and in the increase of the effective value of $\alpha(N, q)$.

In addition to fibers, density correlations were also studied for rectangles of smaller aspect ratios $(20 / 5)$, and isotropic discs. For the flocculation model it was found that the power-law type of form for $G(r)$ remains, but emerges for smaller values of $p$ if the objects are more isotropic. For the rejection model, it was found that correlations become so weak that a power law form is in practice not observed.

It is interesting to examine the results above in light of the experiments on paper a real mass density measurements discussed in the Section 1, with $G(r) \propto r^{-037}$, and the cutoff increasing with decreasing basis weight. The flocculation model can give similar values of $\alpha \approx 0.37$ for experimentally relevant values of $N$, by suitably adjusting $p$. However, the range of the power law in this case does not vary with areal mass density as in the experiment. There are thus at least two possibilities of obtaining longer range correlations such as in the low mass density samples. The first is that such paper comprises small clusters between which there is an effective interaction giving rise to such correlations as explained in Ref. [33]. The second is that the emergence of longer ranged correlations can be thought to arise from larger clusters with no inter-cluster orrelations, but with internal correlations of power law type, with $\alpha \approx$ 0.37 .

\subsubsection{The pair distribution function}

An important function that is closely related to $G(r)$ and yields information about spatial correlations in the deposits, is the pair distribution function $\Omega(r)$ of the centers of mass of the deposited objects. It is defined by
$\Omega(r) \mathrm{d} r$

$$
=\left[\begin{array}{c}
N \\
2
\end{array}\right]^{-1} \times\left\langle\sum_{\vec{x}_{0}} \Delta N_{\mathrm{CM}}\left(\vec{x}_{0}\right) \Delta N_{\mathrm{CM}}\left(\vec{x}_{0}+\vec{x}\right)\right\rangle,
$$

where $\Delta N_{\mathrm{CM}}(\vec{x})$ is the number of centers of masses within the area element $\Delta A(\vec{x})$ around the position vector $\vec{x}$, and $r=|\vec{x}|$ (brackets denote configuration averaging). For the case of a uniformly random network, there exists an exact result derived by Ghosh [39] as

$$
\begin{aligned}
& \Omega_{\mathrm{a}}(r)= \\
& \left\{\begin{array}{l}
\left(4 r / L^{4}\right)\left[\pi L^{2} / 2-2 r L+r^{2} / 2\right], \quad \text { for } 0 \leq r \leq L ; \\
\left(4 r / L^{4}\right)\left[L^{2}(\arcsin (L / r)-\arccos (L / r))\right. \\
\left.+2 L \sqrt{r^{2}-L^{2}}-\frac{1}{2}\left(r^{2}+2 L^{2}\right)\right], \quad \text { for } L \leq r \leq \sqrt{2} L,
\end{array}\right.
\end{aligned}
$$

where $L$ is the linear system size. For the case of the flocculation model close to $p=0$, this result has been generalized based on the approximation that $\Omega(r)$ can be written as a weighted sum of contributions coming from the inner correlations in single clusters, and uniform random deposition of such clusters [29]. In particular,

$\Omega(r)=\omega_{1}(N, p) g(r)+\omega_{2}(N, p) \Omega_{\mathrm{a}}(r, L)$,

where

$$
\begin{aligned}
& g(r) \\
& =\left\{\begin{array}{cc}
\left(30 r / 42 R^{5}\right)\left(3 r^{3}-6 R r^{2}+4 R^{3}\right), & \text { for } 0 \leq r \leq R ; \\
\left(30 r / 42 R^{5}\right)(2 R-r)^{3}, & \text { for } R \leq r \leq 2 R, \\
0, & \text { for } 2 R \leq r
\end{array}\right.
\end{aligned}
$$

The quantities $\omega_{1}(N, p)=n_{\mathrm{c}} N_{\mathrm{c}}\left(N_{\mathrm{c}}-1\right) /[N(N-$ $1)]$, and $\omega_{2}(N, p)=1-\omega_{1}(N, p)$, where $n_{\mathrm{c}}$ is the number of effective clusters in the network. This is treated as a fitting parameter thus fixing $N_{\mathrm{c}}$, the number of fibers in a given cluster, by $N_{\mathrm{c}}=N / n_{\mathrm{c}}$. 
In Fig. 10 we show comparisons between the theoretically calculated and numerically obtained functions for the flocculation model, for values of $N$ well below percolation (above percolation $\Omega(r)$ rapidly approaches Eq. (11)). The position of the peak near the origin reflects the average cluster size in the system. The peak vanishes in the limit of large $N$.

The behavior of $\Omega(r)$ for the rejection model is quite different. For the case of needles, the series of functions are shown in Fig. 11(a). Within the numerical accuracy, $\Omega(r)$ was found to be indepen- dent of $q$. This can be understood by the fact that widthless needles can be deposited arbitrary closely together (when aligned), and thus the distribution of centers of masses very closely follows that of a uniformly random deposit. However, the situation changes for the case where the deposited objects have a finite area [40]. In Fig. 11(b) we show a series of distribution functions for $2 \mathrm{D}$ spheres of radius $R=0.5$. Approaching the RSA limit, the increasing penalty for overlap causes the distribution function to be sharply cut off at $r_{\mathrm{c}}=$ $2 R$. This behavior can be approximated by writing
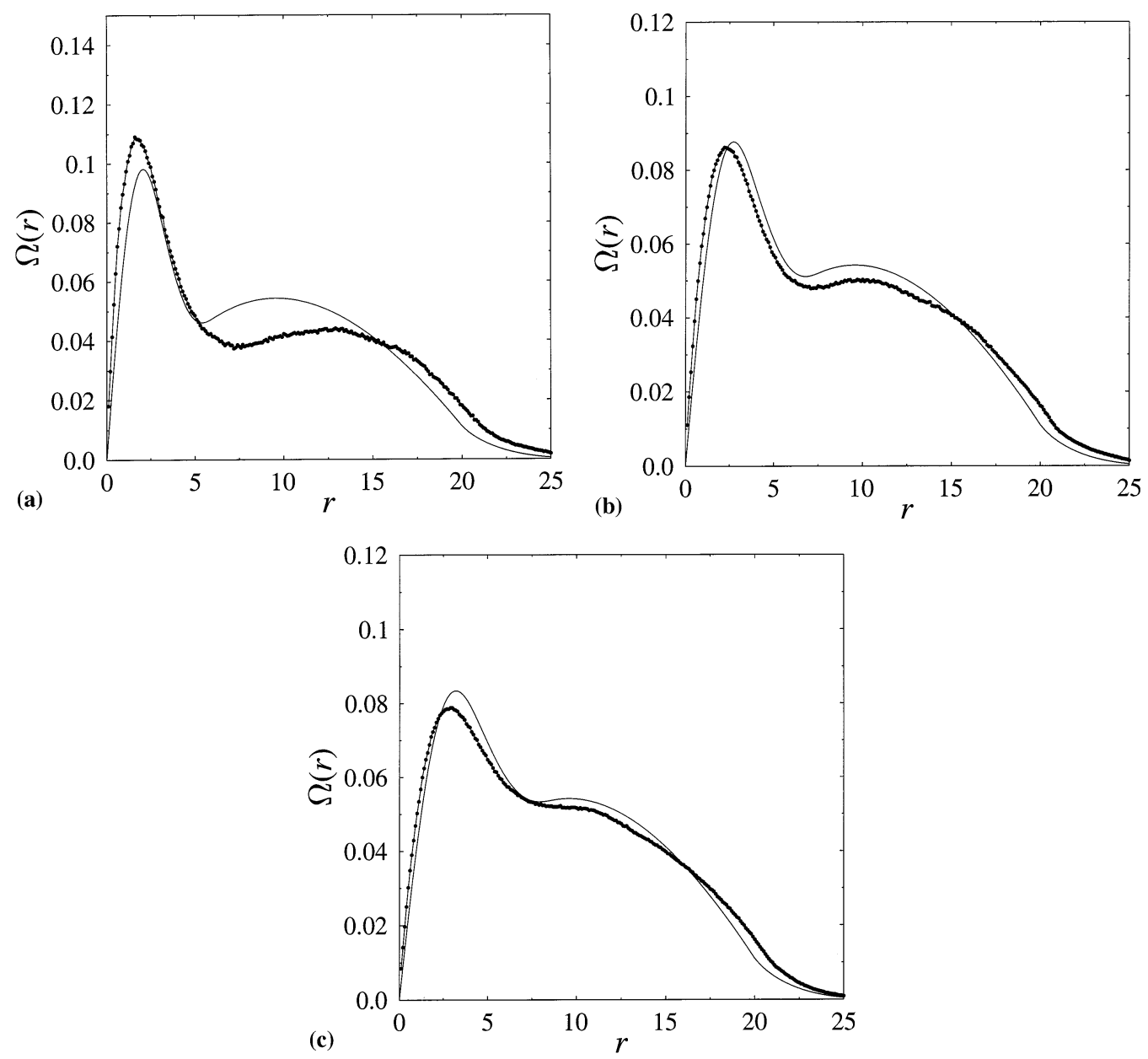

Fig. 10. Comparison between analytic (solid lines) and simulated (dotted lines) pair distribution functions for the flocculation model ( $p=0.001$ ) with (a) $N=250$; (b) $N=500$, and (c) $N=750$. Here $L=20, \lambda=1$ and $\omega=1 / 4$. 

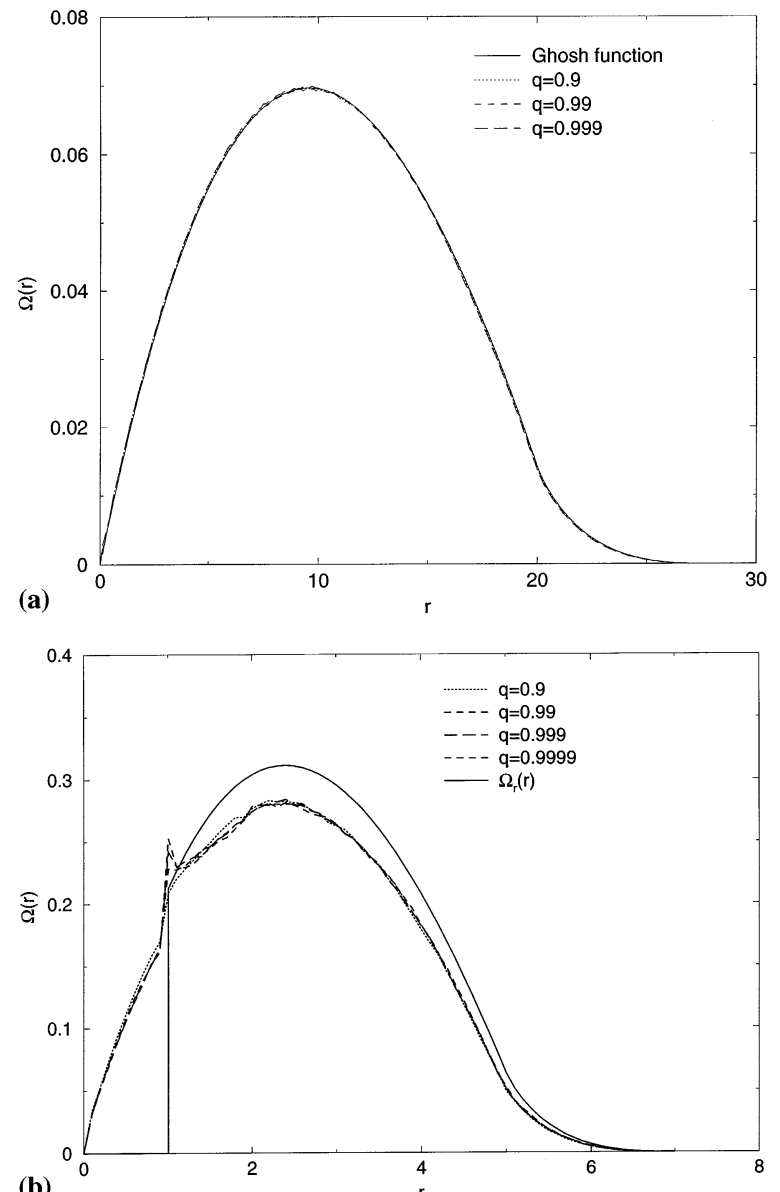

(b)

Fig. 11. (a) Pair distribution functions as calculated numerically for the rejection model with $q=0.9,0.99$, and 0.999 . The solid line denotes the exact Ghosh function of Eq. (11) with $L=20$. In the present case of widthless needles of unit length the results are independent of $q$ within the accuracy of the data. (b) Corresponding functions for the case of circles of unit diameter. The solid line denotes the analytic approximation of Eq. (14). System size $L=5$.

$\Omega_{\mathrm{r}}(r)=C \Omega_{\mathrm{a}}(r) \theta\left(r-r_{\mathrm{c}}\right)$,

where $\theta(r)$ is the step function, and $C$ a normalization constant that depends on $L$ and $r_{\mathrm{c}}$. The analytic approximation is also plotted in Fig. 11(b). We note that it does not reproduce the strong peak just beyond $r_{\mathrm{c}}$, which is due to the fact that the objects tend to be as close as possible when the RSA limit is approached.

\section{3D fiber deposition model}

In this section we examine the growth of $3 \mathrm{D}$ fiber networks out of fibers that have finite flexibility [5]. This is motivated by the fact that fiber network will be truly three-dimensional only after pores start to form inside the structure. We consider here structures such as real paper, so that the fibers are primarily oriented along planes perpendicular to the direction of deposition in the growth model.

We start with substrates which form a square lattice in $2 \mathrm{D}$, or a linear array of sites in $1 \mathrm{D}$. The particles deposited are initially straight fibers, typically of unit width and thickness $\left(w_{\mathrm{f}}=\right.$ $\left.t_{\mathrm{f}}=1\right)$. Emphasis is given to fiber lengths $L_{\mathrm{f}} \gg 1$. Fibers are positioned at random in such a way that the local coverage $c$ (number of fibers covering each lattice site) is an integer. Periodic boundary conditions are applied in the plane of deposition.

Non-trivial porous structures arise when the fibers have a non-zero stiffness. A bending fiexibility $T_{\mathrm{f}}$ of the fibers is defined through a constraint on the height of local 'steps' that the fibers can form:

$\left|z_{i}-z_{j}\right| \leq T_{\mathrm{f}}$,

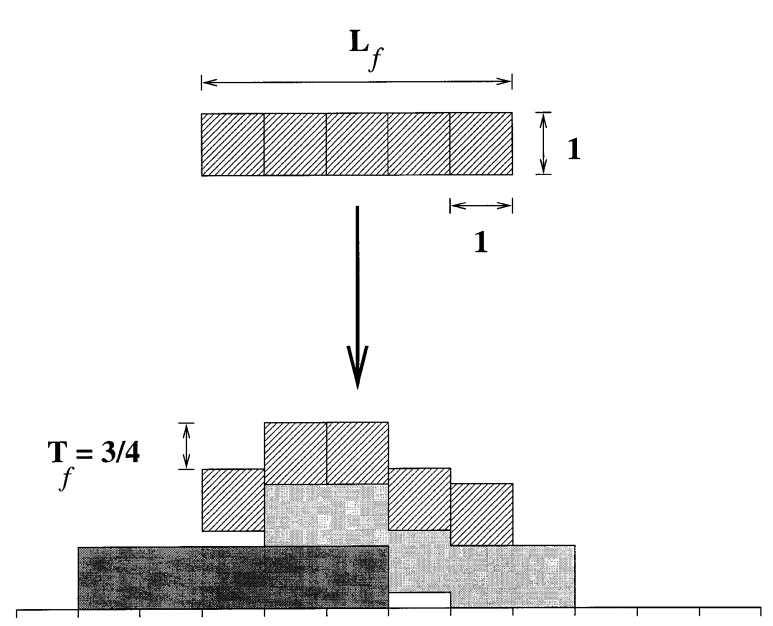

Fig. 12. Deposition of a fiber with length $L_{\mathrm{f}}=5$ and flexibility $T_{\mathrm{f}}=3 / 4$. 


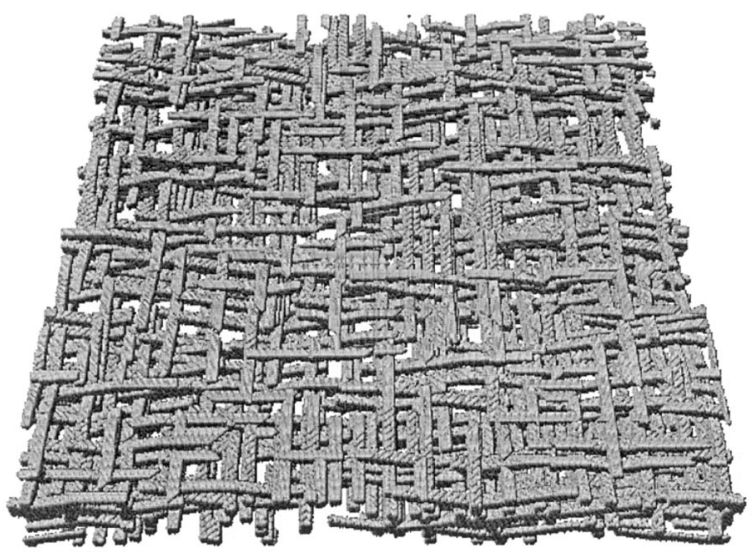

Fig. 13. An example of a 2D fiber network constructed with the $3 \mathrm{D}$ fiber deposition model. The porosity of the network is about 0.83 .

where $z_{i}$ and $z_{j}$ are the elevations of the top surface of the fiber above any two nearest neighbor cells $i$ and $j$ covered by the fiber (cf. Fig. 12). In the simulations fibers are deposited one at a time with no in-plane correlations. The fibers are placed down directly on the already formed network and deformed to lie as low as possible while still obeying Eq. (15). Fig. 13 shows what the disordered network looks like in 2D. Formation of pores inside a one-dimensional network is illustrated in Fig. 14.
Characterizing a stochastic structure with the aid of just a few parameters is a highly nontrivial task. It turns out, however, that many of the statistical properties of the network structure can be expressed as a function of the coverage $c$ and a dimensionless flexibility number $F$ [5], related to previously introduced fiexibility, as follows:

$F=T_{\mathrm{f}} w_{\mathrm{f}} / t_{\mathrm{f}}$

The flexibility dependence of several quantities related to the pore structure has been studied in Refs. [5,41,42].

The model we are considering here is similar to many growth models and particularly to the Vold model $[43,44]$. As the local interactions in our fiber deposition model are replaced with $F$ (or $T_{\mathrm{f}}$ ) we are ignoring many effects present in real sedimentation processes. These include clustering or flocculation of the fibers as discussed earlier in this review, and reordering along the surface because of hydrodynamic or gravitational forces [45]. Moreover, the simple bending rule does not accurately describe the hydrostatic pressure [46] and the underlying network does not change when new fibers are deposited. It has also been shown that the model does not reproduce the surface roughness properties of real paper sheets [47]. Ordinary paper sheets are usually compressed mechanically. Such effects are hard to
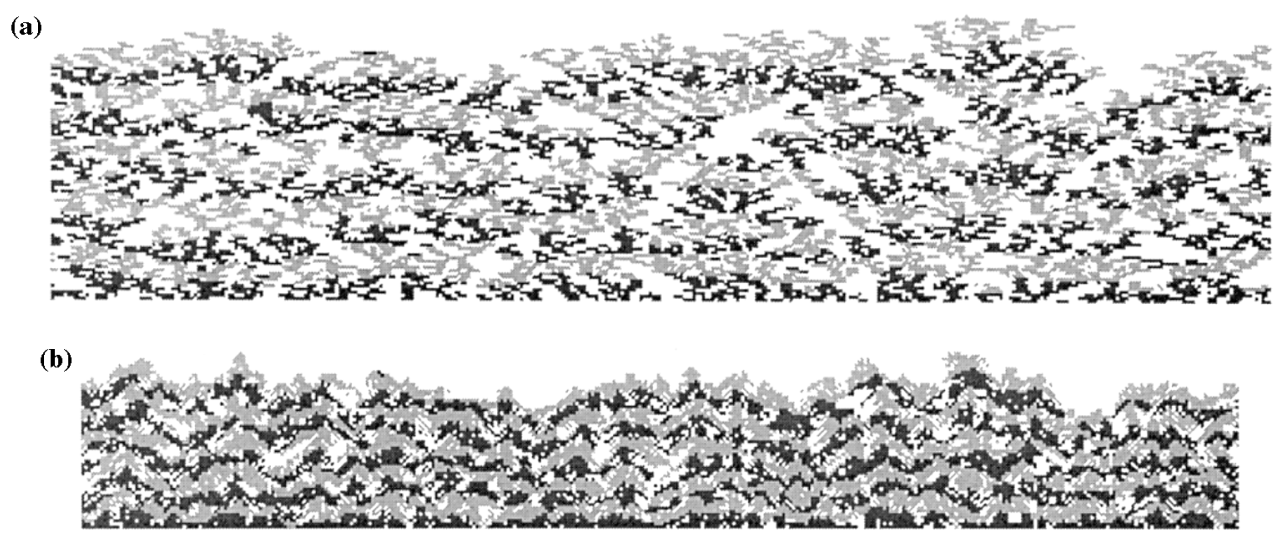

Fig. 14. Typical fiber networks with (a) $T_{\mathrm{f}}=0.1$ and (b) $T_{\mathrm{f}}=1.0$. The system size $L=500$ and the fiber length $L_{\mathrm{f}}=5$. The white areas are pores and the grey areas indicate the fibers. The number of fibers is 5000 and each layer marked by either of the two shades of grey contain 500 fibers. 
take into account as they depend on the actual initial spatial structure of the deposit itself [46]. The distribution of mass per unit area is Poissonian, unlike in real paper which has nontrivial spatial correlations, as discussed in the Introduction [13]. Such weak correlations should however be of negligible importance with respect to self-averaging transport properties which we will discuss in Section 3.2 below.

\subsection{Statistical properties of networks}

In the following we discuss the differences between 2- and 3D fiber networks. We consider the in-plane percolation properties of deposits, the connectivity of the networks (where connectivity means fiber-fiber contacts per fiber) and pore statistics in 3D networks. These issues are well understood in strictly 2D networks [48], whereas analytical models [38,49] for fiber networks with finite thickness are not accurate due to the fact that contacts between fibers are controlled by the random pore structure.

At low coverage $c$ the fiexibility $F$ is not a relevant quantity to describe the in-plane properties of a planar network. Instead, these properties should be controlled by $c / \eta_{\mathrm{c}}$, where $\eta_{\mathrm{c}}$ is the percolation threshold of the corresponding 2D network [50,51]. In our model the computed threshold is given by

$\eta_{\mathrm{c}} \approx 5.7 / L_{\mathrm{f}}$

for long and flexible fibers $\left(L_{\mathrm{f}} \geq 30-40\right)$. For short fibers

$\eta_{\mathrm{c}} \approx 4 / L_{\mathrm{f}}, \quad$ for $L_{\mathrm{f}}=7$ and $T_{\mathrm{f}}>1$;

$\eta_{\mathrm{c}} \approx 3.5 / L_{\mathrm{f}}, \quad$ for $L_{\mathrm{f}}=3$ and $T_{\mathrm{f}} \geq 5$

In the latter case the non-zero width $w_{\mathrm{f}}$ lowers the percolation threshold at high $T_{\mathrm{f}}$ [5]. A random walk argument suggests that in the opposite limit, i.e. when $T_{\mathrm{f}} \rightarrow 0$, the radius $r$ of a cluster with $N$ fibers goes like $r \sim L_{\mathrm{f}} \sqrt{N}$, and therefore the outof-plane thickness of a percolating cluster is roughly given by $t_{\mathrm{f}}\left(L / L_{\mathrm{f}}\right)^{2} \gg L$. Thus, $\eta_{\mathrm{c}}$ diverges and no longer characterizes its structure appropriately and the network becomes three-dimensional. The crossover between the flexible and stiff fiber percolation behavior occurs at $T_{\mathrm{f}} \sim 4 t_{\mathrm{f}} / L_{\mathrm{f}}$, since the maximum vertical deflection per unit length of a fiber is $T_{\mathrm{f}}$.

With increasing coverage $c$, a cross-over from a 2D network to a pore-controlled 3D structure occurs at $c=c_{0} \approx 1+2 F$. At $c>c_{0}$ 3D pores develop in the network, as opposed to the $2 \mathrm{D}$ vacancies. In the asymptotic high-coverage region the flat substrate no longer has any effect. The pore number $p_{\mathrm{n}}$ i.e. the number of missing fiberfiber contacts, increases linearly with coverage as

$p_{\mathrm{n}}=p_{\infty}^{\prime}\left(c-c_{0}\right)$

Here $p_{\infty}^{\prime}$ is the slope of pore number relative to the coverage. The expression

$p_{\infty}^{\prime}=1-n_{\infty} \simeq \frac{1-\exp (-2 F)}{2 F}$,

gives a surprisingly good match to the simulated data, where $n_{\infty}$ is the fraction of fiber surface in contact with others, i.e. the coordination number [41].

Various measurements [41,42] yield that the pore-height distribution is essentially exponential,

$G(h)=h_{0}^{-1} \exp \left(-h / h_{0}\right)$

with $h_{0} \sim 1 / n_{\infty}$. The same also holds for the distribution of pore areas:

$P(A) \sim \exp \left(-A / A_{0}\right)$,

with $A_{0} \sim 1 / F$.

Given that the pore heights and cross-sectional areas are only weakly correlated, the average pore volume $\bar{V}$ is close to the product of the average area and height of the pores. One obtains $\bar{V} \approx 1.5 /$ $n_{\infty}^{2}$. The average surface area $\bar{S}$ of the pores becomes $\bar{S}=2 \bar{A}+4 \bar{h} \sqrt{\bar{A}}$, and the surface area of the pores per unit volume $\bar{S} p_{\infty}^{\prime} \approx 2\left(3+\sqrt{n_{\infty}}\right)(1-$ $\left.n_{\infty}\right) / n_{\infty}^{1.5}$ in the asymptotic high coverage region. The quantity $S$ has a non-trivial weak maximum as a function of $F$ [41], which has e.g. implications for gas adsorption in porous networks.

\subsection{Transport through networks}

The mechanical and transport properties of disordered materials depend on their geometry. This 


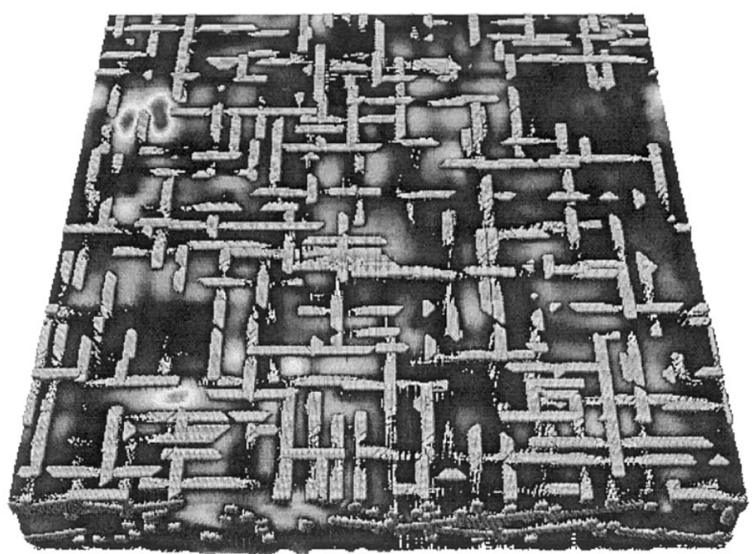

Fig. 15. The in-plane velocity field of the fiber network shown in Fig. 13. Lighter shades of grey indicate high fluid velocities.

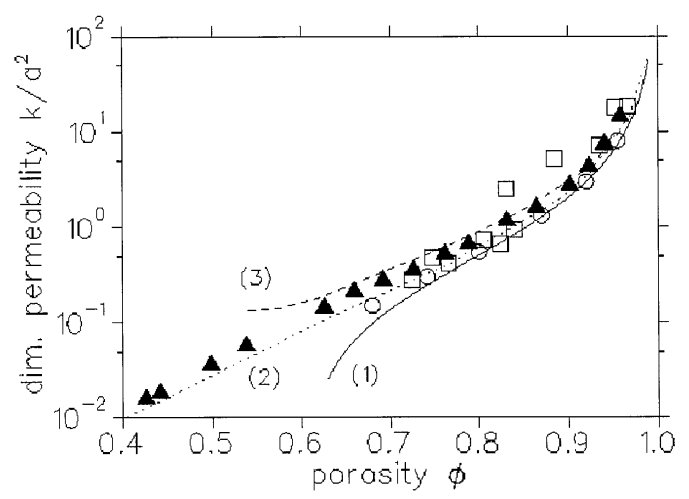

Fig. 16. The simulated non-dimensional permeabilities $k / a^{2}$ as a function of porosity (black triangles). White boxes and circles show the experimental results for fibrous filters [69] and compressed fiber mats [70,69], respectively. Curve 1 is an analytical prediction for cubical lattice geometry [69], curve 2 is the simulated result with fcc geometry [71] and curve 3 shows the prediction given by the Kozeny-Carman Eq. (27).

makes modelling difficult since one may need a complete characterization of the geometry of the medium including higher-order statistics [52]. Recently, developed methods include variational bounds, for which 2 nd and 3rd order correlation functions are needed [53], effective medium techniques [54-57] and percolation-type ideas [58,59]. Simulated fiber networks may enable one to study transport properties of disordered media efficiently $[28,41,60]$.
A good example in which the geometry of the pore system plays a crucial role is permeability. If the pore system can be assumed to be well-connected then the capillary approximation should hold. That means that the permeability depends only on the effective hydraulic radius of a typical pore and the contact angle of the fluid phase $[61,62]$. The capillary approximation is not useful when the pore system consists of large cavities that are connected through narrow pore throats. Other ideas may then be useful, e.g. trying to relate the distribution of the throats to the percolation threshold in connectivity $[58,59,63]$.

For 3D fiber networks, a variational bound for the hydraulic conductivity $g$ in the out-of-plane direction can be estimated by using the typical pore structures [41]. The hydraulic conductance $g_{\mathrm{h}}$ of an individual pore is given by $[58,59]$

$g_{\mathrm{h}}(A, h)=\frac{A^{2}}{8 \pi h \mu}$,

where $A$ is again the pore area, $h$ the pore height, and $\mu$ the fluid viscosity. Using a variational trial solution, the pore statistics $G(h), P(A)$ (Section 3.1 , and the approximation that the pore height and area are independent, one gets in the asymptotic high coverage region

$g \sim(1-\exp (-2 F)) / F^{3}$

This is a rapidly and monotonically decreasing function of $F$.

Log-normal distributions for pore sizes are often observed in experiments $[64,65]$, in contrast to the exponential distributions given above. However, such observations are based on 'thin' sheets of paper for which the capillary approximation used may not be appropriate [64]. One can illuminate the differences caused by differently structured disordered media by considering direct lattice-Bolzmann simulations of fluid flow in $3 \mathrm{D}$ networks (cf. Fig. 15) [28]. Assuming creeping flow, and Darcy's law [66-68]

$\mathbf{q}=-\frac{k}{\mu} \nabla p$

the permeability coefficient $k$, a measure of the fluid conductivity through the pore system, can be simulated. Here $\mathbf{q}$ is the flux of the fluid through 
the porous medium, $\mu$ is the viscosity of the fluid and $p$ is the fluid pressure.

The simulated dimensionless permeabilities in the in-plane direction are shown as a function of porosity $\phi$ in Fig. 16, where each black triangle represents the simulated permeability of one single fiber network [28]. The fiber network resembles fibrous filters, for which experimental permeability results [69] are shown in Fig. 16 with open squares. The simulated points are seen to agree well with the experimental set of data. The open circles in Fig. 16 correspond to experimental data for compressed random fiber mats $[69,70]$.

The simulated permeabilities are also compared with analytical and numerical results for disordered fiber networks. Curve (1) in Fig. 16 shows an analytical prediction of permeability for a cubic lattice model, namely $k / a^{2}=-\ln (1-\phi)-$ $0.931+O(1 / \ln (1-\phi))[69]$. Curve (2), which has been computed numerically for a regular fiber array with a face-cantered-cubic (fcc) geometry [71], shows that disorder leads to a larger permeability of the network [69]. In the same figure we also present a fit to the data which utilizes the semiempirical Kozeny-Carman equation [66]

$k=\phi^{3} / c S^{2}$,

where $S$ is the specific surface area of the porous medium and $c$ is the so called 'Kozeny constant', which is known to be porosity dependent for fibrous media [72]. Curve (3) in Fig. 16 uses the experimental fit for the Kozeny constant $c=$ $3.5 \phi^{3}\left[1+57(1-\phi)^{3}\right] /(1-\phi)^{1 / 2}$, which has been suggested for fibrous materials for porosities higher than 0.6 [70,72]. These comparisons show that the simulated networks can be indeed be used to study transport properties.

\subsection{Kinetic roughening of growing networks}

An interesting question concerns the asymptotic properties of the growing network in the $3 \mathrm{D}$ deposition model. This can be discussed in terms of the theory of kinetic roughening of growing interfaces $[9,73]$. In the following we outline the physics of the surface roughness for the particular example of porous fiber networks grown on a $1 \mathrm{D}$ substrate [74].
For many cases of interest, the kinetics of growing surfaces can be described by the following type of equation of motion, called the Kardar-Parisi-Zhang (KPZ) equation [75]:

$\frac{\partial h(\vec{r}, t)}{\partial t}=\nabla^{2} h(\vec{r}, t)+\frac{\lambda}{2}|\nabla h(\vec{r}, t)|^{2}+\eta(\vec{r}, t)+v_{0}$

Here $h(\vec{r}, t)$ is a single-valued height variable that defines the position of the interface, $\eta(\vec{r}, t)$ is a Gaussian (white) noise term and $v_{0}$ is a constant. The nonlinear term proportional to $\lambda$ arises in the growth process if the local velocity of the growing surfaces depends on the tilt. In fact, in the 3D fiber deposition model this is easily seen to be the case when the fibers are not infinitely flexible. This process is thus intimately related to the formation of pores.

In the present case we are now only interested in the free top surface of the network. It is defined by the set of local height variables $h(x, t)$ at each lattice site $x$. Thus, the curve defined by $h(x, t)$ is a single-valued function so that overhangs in the surface structure are ignored.

In the growth simulations time is defined in terms of coverage $c$ which is the amount of mass deposited per unit substrate length. Since the mass of a fiber is $L_{\mathrm{f}}$, the deposition of $N$ fibers on a 1D lattice of size $L$ takes $t=N\left(L_{\mathrm{f}} / L\right)$ time steps. If the number of particles deposited in a unit time is kept constant, the spatial average of the surface height, $\bar{h}(t)=\Sigma_{i=1}^{L} h\left(x_{i}, t\right) / L$, grows linearly in time. This assumes that the porosity is constant in the new deposit that is being formed.

A convenient way to characterize the surface roughness is through the average interface width $w^{2}(L, t)=\left\langle\overline{[h(\vec{r}, t)-\bar{h}(t)]^{2}}\right\rangle$,

where $\bar{h}(t)$ denotes the spatial average of $h(\vec{r}, t)$, and the brackets denote average over the noise. The width obeys the scaling form

$w(L, t)=L^{\chi} f\left(t / L^{z}\right)$,

where the scaling function $f(y)$ behaves as

$f(y) \sim\left\{\begin{array}{c}y^{\beta}, \text { for } y \ll 1 ; \\ \text { const., for } y \gg 1\end{array}\right.$ 
The relevant set of scaling exponents are the dynamical exponent $z$, the roughness exponent $\chi$ and the growth exponent $\beta$, which are linked by $\chi=z \beta$, and the exact relation $\chi+z=2$. For a $1 \mathrm{D}$ interface, the stationary probability distribution associated with Eq. (28) is known, and the exponents are given by $\beta=1 / 3, \chi=1 / 2$, and $z=3 / 2$. In the case of the $1 \mathrm{D}$ substrate, the scaling exponents were determined from the surface width and from the relevant height-height correlation functions $[9,76]$. The results are in excellent agreement with the expected KPZ universality class, with $\beta=0.338 \pm 0.003$, and $\chi=0.49 \pm 0.02$.

The key factor determining the universality class of growth in the model is the flexibility parameter $T_{\mathrm{f}}$. In the limit where $T_{\mathrm{f}} \rightarrow \infty$, the local surface height just depends on the number of fibers at a site, and the model is equivalent to the so-called (trivial) random deposition (RD) case, with $\beta=1 / 2$ and $\chi$ undefined [9]. For $T_{\mathrm{f}}<\infty$, however, the finite rigidity of the fibers leads to nontrivial correlations that manifest themselves as bulk defects (pores) in analogy to some other surface deposition models [73,77]. This makes it possible to extract the crossover time $t_{\mathrm{c}}$ from RD to the KPZ regime due to the formation of pores as follows. Since each deposition event simultaneously fills $L_{\mathrm{f}}$ sites, the width in the RD regime is

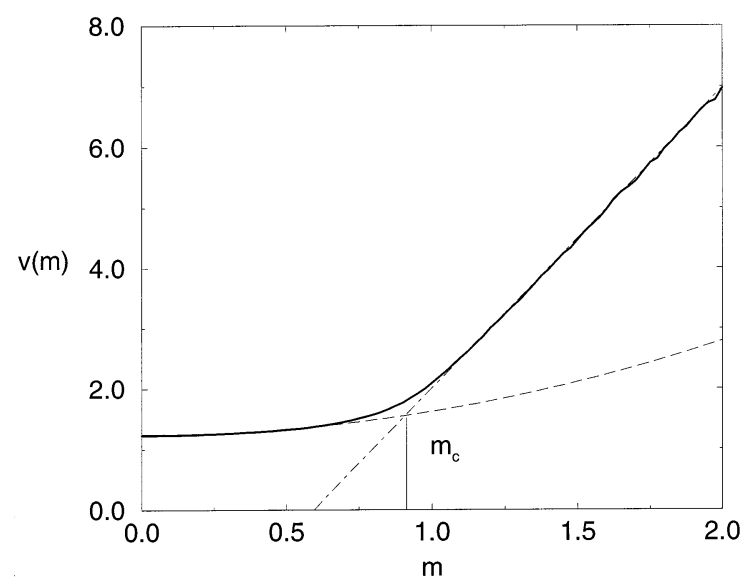

Fig. 17. Typical data for $v(m)$ (solid line) as a function of the tilt $m$ of the substrate as $L=2500, T_{\mathrm{f}}=1.0$ and $L_{\mathrm{f}}=11$. The dashed line is a fit of the form of Eq. (34) and the dot-dashed line is calculated from Eq. (35). Their intersection defines $m_{\mathrm{c}}$.
$W_{\mathrm{RD}}(t) \approx\left(t / L_{\mathrm{f}}\right)^{1 / 2}$

In the $\mathrm{RD}$ regime, the surface has no spatial correlations, and hence $w_{\mathrm{RD}}$ is a measure also of the nearest neighbor height differences. The RD regime ends when these become of the order $T_{\mathrm{f}}$, such that the finite flexibility of the fibers is felt. Setting $w_{\mathrm{RD}}\left(t_{\mathrm{c}}\right) \approx T_{\mathrm{f}}$, it was concluded that

$t_{\mathrm{c}} \approx T_{\mathrm{f}}^{2} L_{\mathrm{f}}$

Note, in particular, that for any non-zero fiexibility an extended RD regime appears for sufficiently long fibers, $L_{\mathrm{f}} \gg 1 / T_{\mathrm{f}}^{2}$.

The average KPZ growth velocity can be written in terms of the macroscopic tilt $m \equiv \nabla h$ to the network as

$v(m)=v(0)+\frac{\lambda}{2} m^{2}$

Thus, by measuring $v$ as a function of $m$ one can get an estimate for $\lambda$ as a function of $T_{\mathrm{f}}$. Through the relation $v(m)=1 / \rho(m)$ this also provides information on the deposit density $\rho$ [77].

Fig. 17 shows the typical behavior of $v(m)$ for both small and large values of $m$. It demonstrates that $v(m)$ has two regimes. For small values of $\mathrm{m}$, it follows Eq. (34) as shown by the dashed line, while for large values of $m$ the dependence becomes linear. We can define the crossover tilt $m_{\mathrm{c}}$ between these two regions by the point of intersection of Eq. (34) and the linear fit, as shown in the figure. It turns out that $m_{\mathrm{c}} \approx T_{\mathrm{f}}$.

The linear regime for the growth velocity as a function of tilt evident in Fig. 17 is easy to understand: the fibers tend to conform along the surface according to the amount given by the stiffness parameter $T_{\mathrm{f}}$. An equation for $v(m)$ has been derived in Ref. [74] and reads as

$v(m)=\frac{1}{2}\left(L_{\mathrm{f}}-1\right)\left(m-T_{\mathrm{f}}\right)+2, m>T_{\mathrm{f}}$,

where $v_{\infty} \equiv v\left(T_{\mathrm{f}}=\infty\right)=1$ in our units. The validity of Eq. (35) was verified quantitatively for a system with $L=2500, T_{\mathrm{f}}=1.0$ and $L_{\mathrm{f}}=11$ for which it gives $v(m)=5.0 m-3.0$ as $m>1.0$. This equation is indicated in Fig. 17 as a dotdashed line and it shows perfect agreement with the data. 


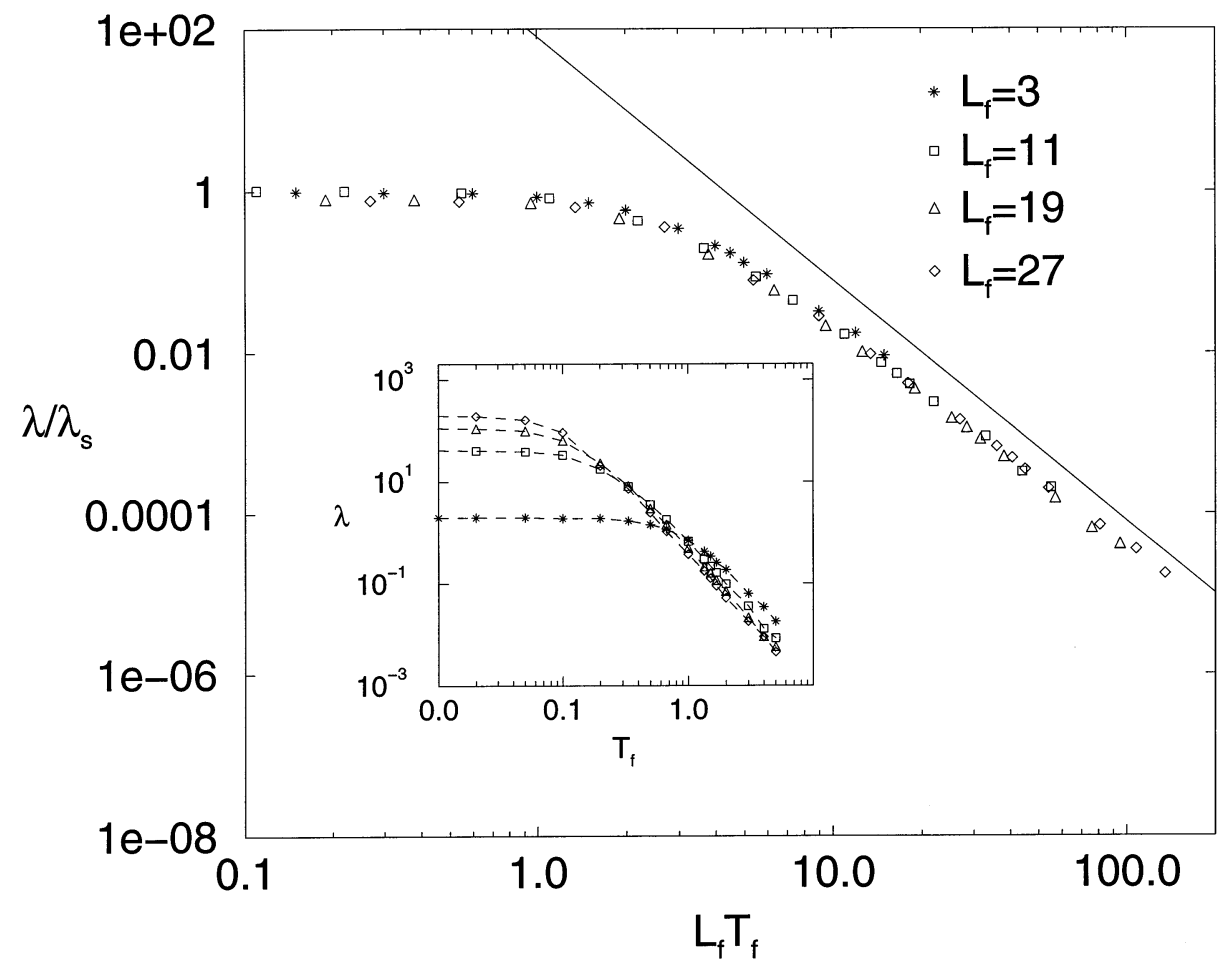

Fig. 18. The ratio $\lambda / \lambda_{\mathrm{s}}$ as a function of $L_{\mathrm{f}} T_{\mathrm{f}}$. This curve was obtained by collapsing four $\lambda\left(T_{\mathrm{f}}\right)$ curves corresponding to $L_{\mathrm{f}}=3$, 11 , 19 and 27 (shown in the inset) into a single curve. In the simulations the number of layers deposited was 500 and the number of runs was 10 . The solid line obeys $\lambda / \lambda_{s} \sim\left(L_{\mathrm{f}} T_{\mathrm{f}}\right)^{-3}$.

For small tilts and with $m \ll T_{\mathrm{f}}$, the growth rate $v(m)$ is of the form of Eq. (34). The nonlinearity parameter $\lambda$ can be then determined by leastsquares fitting. Fig. 18 shows how $\lambda$ depends on the fiber fiexibility $T_{\mathrm{f}}$ for four fiber lengths $L_{\mathrm{f}}$ when the system size is $L=2500$. The original data (shown in the inset) collapse into a single scaling curve by multiplying $T_{\mathrm{f}}$ by $L_{\mathrm{f}}$ and dividing $\lambda$ by $\lambda_{\mathrm{s}} \equiv \lambda\left(T_{\mathrm{f}}=0\right)$ corresponding to a stiff fiber. In this limit, $\lambda_{s}$ was calculated separately as a function of $L_{\mathrm{f}}$ and the phenomenological scaling form $\lambda_{\mathrm{s}}=c\left(L_{\mathrm{f}}-1\right)^{2}$ was obtained where $c=$ $0.40 \pm 0.03$.

According to Fig. 18, in the case of small tilts $\lambda$ has two regimes roughly separated by $L_{\mathrm{f}} T_{\mathrm{f}} \approx 1$. As $L_{\mathrm{f}} T_{\mathrm{f}} \ll 1, \lambda \approx \lambda_{\mathrm{s}}$ and it does not depend on $T_{\mathrm{f}}$. On the other hand, in the $L_{\mathrm{f}} T_{\mathrm{f}} \gg 1$ regime there is clearly an inverse power law behavior as a function of $T_{\mathrm{f}}$. When the running exponent method was applied to $\lambda / \lambda_{\mathrm{s}}$ corresponding to the case of $L_{\mathrm{f}}=27$, it gave for the value of the exponent of this power law $-2.8 \pm 0.4$ implying that $\lambda / \lambda_{\mathrm{s}} \sim$ $\left(L_{\mathrm{f}} T_{\mathrm{f}}\right)^{-2.8}$. Thus, we can summarize the scaling behavior of $\lambda$ as

$\lambda \sim\left\{\begin{array}{c}\left(\mathrm{L}_{\mathrm{f}}-1\right)^{2}, \text { for } L_{\mathrm{f}} T_{\mathrm{f}} \ll 1 ; \\ L_{\mathrm{f}}^{-1} T_{\mathrm{f}}^{-2.8 \pm 0.4}, \text { for } L_{\mathrm{f}} T_{\mathrm{f}} \gg 1\end{array}\right.$

We note that this form correctly satisfies the RD limits of the model where $\lambda \rightarrow 0$ for either $L_{\mathrm{f}}=1$ or $T_{\mathrm{f}} \rightarrow \infty$.

To explain Eq. (36) a simple scaling theory was developed in Ref. [74]. There it was shown that

$\lambda \sim\left\{\begin{array}{c}\mathrm{L}_{\mathrm{f}}^{2}, \text { for } L_{\mathrm{f}} T_{\mathrm{f}} \ll 1 ; \\ L_{\mathrm{f}}^{-1} T_{\mathrm{f}}^{-3}, \text { for } L_{\mathrm{f}} T_{\mathrm{f}} \gg 1\end{array}\right.$ 
These limits agree well with the numerical result in Eq. (36). The solid line in Fig. 18 shows $\lambda / \lambda_{\mathrm{s}} \sim\left(L_{\mathrm{f}} T_{\mathrm{f}}\right)^{-3}$.

To conclude, the surface roughening of porous fiber networks in one substrate dimension obeys KPZ type of scaling. It has been possible to establish scaling arguments to explain the scaling of the KPZ nonlinearity $\lambda$ with $T_{\mathrm{f}}$ that is on the other hand related to the formation of pores. These arguments make it seem natural that even higher-dimensional variants are in the KPZ universality class.

\section{Summary and discussion}

To summarize, this review discusses 2- and 3D deposition models of fibers and other extended objects. In 2D a simple parametrization of effective fiber-fiber interaction is capable of producing a variety of phenomena interpolating between restricted sequential adsorption and single cluster growth. Similarly, in 3D a simple growth rule captures the essential physics in the formation of porous deposits.

For 2D models, our emphasis was on two phenomena, namely percolation and spatial correlations. The percolation threshold of the 2D flocculation model shows an interesting minimum as a function of the interaction parameter $p$, whereas no such minimum was found for the rejection model.

The spatial correlations found in the networks are well approximated by a power-law type form in a certain length scale regime, a feature also observed in the mass density distribution of real laboratory-made paper sheets. However, the range of correlations found in real paper cannot be reproduced by the simple deposition models considered here. To this extent, more elaborate models are needed, such as, for example, making the acceptance probability of the deposition attempt of a single fiber dependent on its neighbourhood [78,79] might give rise to power-law correlations over longer distances.

To complement the analysis of $2 \mathrm{D}$ networks, the properties of a $3 \mathrm{D}$ deposition model were discussed. In particular, the interesting questions concerning the structure of the pore space and the geometric properties of the top surface were addressed. As a practical application of the model, it has been demonstrated that such a simple deposition model can be used to quantitatively simulate some of the transport properties of disordered fiber networks, such as permeability. Analysis of the roughening of the top surface reveals that it belongs to the ubiquitous Kardar-Parisi-Zhang universality class.

To conclude, we hope to have demonstrated that simple deposition models can be used to qualitatively and, in some cases, quantitatively understand the properties of two- and three-dimensional disordered networks. However, a better quantitative understanding requires more elaborate microscopic models, an avenue for future work.

\section{Acknowledgements}

We wish to thank E. Hellén, A. Koponen, J. Krug, R.M. Nieminen, K. Niskanen, V. Räisänen, E. Seppälä, J. Timonen, J. Vinnurva, J. Åström for fruitful collaboration, and the Academy of Finland MATRA program for financial support.

\section{References}

[1] J.W. Evans, Rev. Mod. Phys. 65 (1993) 1281.

[2] V. Privman, in: D. Stauffer (Ed.), Annual Reviews in Computational Physics, vol. 3, World Scientific, Singapore, 1995.

[3] (a) G.Y. Onoda, E.G. Liniger, Phys. Rev. A 33 (1986) 715. (b) A. Schmit, R. Varoqui, S. Uniyal, J.L. Brash, C. Pusiner, J. Colloid Interface Sci. 92 (1983) 25. (c) J. Feder, I. Giaever, J. Colloid Interface Sci. 78 (1980) 144.

[4] M. Deng, C.T.J. Dodson, Paper: An Engineered Stochastic Structure, Tappi Press, Atlanta, 1994.

[5] K.J. Niskanen, M.J. Alava, Phys. Rev. Lett. 73 (1994) 3475.

[6] P. Nielaba, V. Privman, Phys. Rev. E 51 (1995) 2022.

[7] N. Ryde, H. Kihira, E. Matijevic, J. Colloid Interface Sci. 151 (1992) 421.

[8] P. Wojtaszczyk, E.K. Mann, B. Senger, J.C. Voegel, P. Schaaf, J. Chem. Phys. 103 (1995) 8285. 
[9] A.-L. Barabási, H.E. Stanley, Fractal Concepts in Surface Growth, Cambridge University Press, Cambridge, 1995.

[10] C.A. Murray, D.G. Grier, Am. Sci. 83 (1995) 238.

[11] (a) M.L. Kurnaz, J.V. Maher, Phys. Rev. E 53 (1996) 978. (b) K.V. McCloud, M.L. Kurnaz, J.V. Maher, Phys. Rev. E 56 (1997) 5768.

[12] S. Schwarzer, Phys. Rev. E 52 (1995) 6461.

[13] N. Provatas, M.J. Alava, T. Ala-Nissila, Phys. Rev. E 54 (1996) R36.

[14] G.E. Pike, C.H. Seager, Phys. Rev. B 10 (1974) 1421.

[15] I. Balberg, N. Binenbaum, Phys. Rev. B 28 (1983) 3799.

[16] P.C. Robinson, J. Phys. A 16 (1983) 605.

[17] I. Balberg, C.H. Anderson, S. Alexander, N. Wagner, Phys. Rev. B 30 (1984) 3933.

[18] P.C. Robinson, J. Phys. A 17 (1984) 2823.

[19] A.L.R. Bug, S.A. Safran, G.S. Grest, Phys. Rev. Lett. 55 (1985) 1896.

[20] I. Balberg, Philos. Mag. B 56 (1987) 991.

[21] D. Laría, F. Vericat, Phys. Rev. B 40 (1989) 353.

[22] S.B. Lee, S. Torquato, Phys. Rev. A 41 (1990) 5338.

[23] U. Alon, I. Balberg, A. Drory, Phys. Rev. Lett. 66 (1991) 2879.

[24] C. Vanneste, A. Gilabert, D. Sornette, Phys. Lett. A 155 (1991) 174.

[25] A. Drory, I. Balberg, B. Berkowitz, Phys. Rev. E 49 (1994) 949.

[26] H.S. Choi, J. Talbot, G. Tarjus, P. Viot, Phys. Rev. E 51 (1995) 1353.

[27] M.D. Rintoul, S. Torquato, Phys. Rev. E 52 (1995) 2635.

[28] A. Koponen, D. Kandhai, E. Hellen, M. Alava, A. Hoekstra, M. Kataja, K. Niskanen, P. Sloot, J. Timonen, Phys. Rev. Lett. 80 (1998) 716.

[29] N. Provatas, M. Haataja, E. Seppälä, S. Majaniemi, J. Åström, M. Alava, T. Ala-Nissila, J. Stat. Phys. 87 (1997) 385.

[30] J. Asikainen, T. Ala-Nissila, unpublished (1999).

[31] J. Åström, unpublished MSc Thesis, Ảbo Akademi, Finland, 1989.

[32] W.J. Boudville, T.C. McGill, Phys. Rev. B 39 (1980) 369.

[33] N. Provatas, T. Ala-Nissila, M.J. Alava, Phys. Rev. Lett. 75 (1995) 3556.

[34] D. Stauffer, A. Aharony, Introduction to Percolation Theory Taylor and Francis, Burgess Science Press, Basingstoke, 1994.

[35] N. Provatas, M. Haataja, E. Seppälä, S. Majaniemi, J. Åström, M. Alava, T. Ala-Nissila, Physica A 239 (1997) 304.

[36] E.L. Hinrichsen, J. Feder, T. Jøssang, J. Stat. Phys. 44 (1986) 793.

[37] R. Vigil, R. Ziff, J. Chem. Phys. 91 (1989) 2599.

[38] (a) J. Gorres, C.S. Sinclair, A. Tallentire, Paperi ja Puu 71 (1989) 54. (b) J. Gorres, P. Luner, J. Pulp Paper Sci. 18 (1992) J127.

[39] B. Ghosh, Calcutta Math. Soc. 43 (1) (1951) 17.
[40] G. Tarjus, P. Viot, Phys. Rev. Lett. 67 (1991) 1875.

[41] E.K.O. Hellén, M.J. Alava, K.J. Niskanen, J. Appl. Phys. 81 (1997) 6425.

[42] K. Niskanen, N. Nilsen, E. Hellen, M. Alava, in: C.F. Baker (Ed.), The Fundamentals of Papermaking Materials, Proceedings of the 11th Fundamental Research Symposium, Cambridge, UK, 22-26 September 1997, Pira International, 1997, pp. 1273-1292.

[43] M.J. Vold, J. Colloid Sci. 14 (1959) 168.

[44] J. Krug, H. Spohn, in: C. Godréche (Ed.), Solids far from Equilibrium: Growth, Morphology, Defects, Cambridge University Press, Cambridge, 1991, pp. 479-582.

[45] I. Pagonabarraga, M. Rubi, Phys. Rev. Lett. 73 (1994) 114.

[46] J.D. Sherwood, H. Van Damme, Phys. Rev. E 50 (1994) 3834.

[47] E. Seppälä, M. Alava, K. Niskanen, Paperi ja Puu 78 (1996) 446.

[48] H. Corte, O.J. Kallmes, Tappi J. 43 (1960) 737.

[49] O.J. Kallmes, H. Corte, G. Bernier, Tappi J. 44 (1961) 519.

[50] S. Kirkpatrick, Rev. Mod. Phys. 45 (1973) 574.

[51] L.M. Schwartz, S. Feng, M.F. Thorpe, P.N. Sen, Phys. Rev. B 32 (1985) 4607.

[52] S. Torquato, Appl. Mech. Rev. 44 (1991) 37.

[53] D.A. Coker, S. Torquato, J. Appl. Phys. 77 (1995) 6087.

[54] R. Hilfer, Phys. Rev. B 44 (1991) 60.

[55] R. Hilfer, Phys. Rev. B 45 (1992) 7115.

[56] E. Haslund, B.D. Hansen, R. Hilfer, B. Nost, J. Appl. Phys. 76 (1994) 5473.

[57] B. Virgin, E. Haslund, R. Hilfer, Physica A 232 (1996) 1.

[58] A.J. Katz, A.H. Thompson, Phys. Rev. B 34 (1986) 8179.

[59] J.R. Banavar, D.L. Johnson, Phys. Rev. B 35 (1987) 7283.

[60] V. Räisänen, M.J. Alava, R.M. Nieminen, J. Appl. Phys. 82 (1997) 3747.

[61] A.E. Scheidegger, The Physics of Flow through Porous Media, University of Toronto Press, Toronto, 1974.

[62] M. Sahimi, Rev. Mod. Phys. 65 (1993) 1406.

[63] L.M. Hirsch, A.H. Thompson, Phys. Rev. E 50 (1994) 2069.

[64] W.C. Bliesner, Tappi 47 (1964) 392.

[65] H. Corte, in: H.F. Rance (Ed.), Handbook of Paper Science, Vol. 2, The Structure and Physical Properties of Paper, Elsevier, Amsterdam 1982, Ch. 6.

[66] J. Bear, Dynamics of Fluids in Porous Media, Dover, New York, 1972.

[67] M. Sahimi, Rev. Mod. Phys. 65 (1993) 1393.

[68] M. Sahimi, Flow and Transport in Porous Media and Fractured Rock, VCH, Weinheim, 1995.

[69] G.W. Jackson, D.F. James, Can. J. Chem. Eng. 64 (1986) 364.

[70] W.L. Ingmanson, B.D. Andrews, R.C. Johnson, Tappi 42 (1959) 840.

[71] J.J.L. Higdon, G.D. Ford, J. Fluid. Mech. 308 (1996) 341. 
[72] S.T. Han, Pulp Paper Mag. Can. 70 (1969) T134.

[73] J. Krug, Adv. Phys. 46 (1997) 139.

[74] J. Vinnurva, M.J. Alava, T. Ala-Nissila, J. Krug, Phys. Rev. E 58 (1998) 1125.

[75] M. Kardar, G. Parisi, Y.C. Zhang, Phys. Rev. Lett. 56 (1986) 889.
[76] T. Ala-Nissila, T. Hjelt, J.M. Kosterlitz, O. Venäläinen, J. Stat. Phys. 72 (1993) 207.

[77] M. Schimschak, J. Krug, Phys. Rev. B 52 (1995) 8550.

[78] F.J. Bafaluy, H.S. Choi, B. Senger, J. Talbot, Phys. Rev. E 51 (1995) 5985.

[79] O. Biham et al., unpublished (1999). 